\title{
Blockade of IL-17 signaling reverses alcohol-induced liver injury and excessive alcohol drinking in mice
}

Jun Xu, ${ }^{1,2}$ Hsiao-Yen Ma, ${ }^{1}$ Xiao Liu, ${ }^{1,2}$ Sara Rosenthal, ${ }^{1}$ Jacopo Baglieri, ${ }^{1,2}$ Ryan McCubbin, ${ }^{1}$ Mengxi Sun, ${ }^{1}$ Yukinori Koyama, ${ }^{1,2}$ Cedric G. Geoffroy, ${ }^{3,4}$ Kaoru Saijo, ${ }^{5}$ Linshan Shang, ${ }^{2}$ Takahiro Nishio, ${ }^{2}$ Igor Maricic, ${ }^{1}$ Max Kreifeldt, ${ }^{6}$ Praveen Kusumanchi, ${ }^{7,8}$ Amanda Roberts, ${ }^{6}$ Binhai Zheng, ${ }^{3}$ Vipin Kumar, ${ }^{1}$ Karsten Zengler, ${ }^{9}$ Donald P. Pizzo, ${ }^{10}$ Mojgan Hosseini, ${ }^{10}$ Candice Contet, ${ }^{6}$ Christopher K. Glass, ${ }^{11}$ Suthat Liangpunsakul,,8,12 Hidekazu Tsukamoto, ${ }^{13,14}$ Bin Gao, ${ }^{15}$ Michael Karin ${ }^{16}$, David A. Brenner, ${ }^{1}$ George F. Koob, ${ }^{17}$ and Tatiana Kisseleva ${ }^{2}$

'Department of Medicine, ${ }^{2}$ Department of Surgery, and ${ }^{3}$ Department of Neurosciences, School of Medicine, UCSD, San Diego, California, USA. ${ }^{4}$ Department of Neuroscience and Experimental Therapeutics, College of Medicine, Texas A\&M University, Bryan, Texas, USA. ${ }^{5}$ Department of Molecular \& Cell Biology, University of California, Berkeley, Berkeley, California, USA. ${ }^{6}$ Department of Neuroscience, Scripps Research Institute, La Jolla, California, USA. ${ }^{7}$ Division of Gastroenterology and Hepatology, Department of Internal Medicine, School of Medicine, Indiana University, Indianapolis, Indiana, USA. ${ }^{8}$ Roudebush VA Medical Center, Indianapolis, Indiana, USA. ${ }^{9}$ Department of Pediatrics, ${ }^{10}$ Department of Pathology, and "Department of Cellular and Molecular Medicine, School of Medicine, UCSD, San Diego, California, USA. ${ }^{12}$ Department of Biochemistry and Molecular Biology, School of Medicine, Indiana University, Indianapolis, Indiana, USA. ${ }^{13}$ Southern California Research Center for ALPD and Cirrhosis, Department of Pathology, Keck School of Medicine, University of Southern California, Los Angeles, California, USA. ${ }^{14}$ VA Greater Los Angeles Healthcare System, Los Angeles, California, USA. ${ }^{15}$ Laboratory of Liver Diseases, National Institute on Alcohol Abuse and Alcoholism, NIH, Bethesda, Maryland, USA. ${ }^{16}$ Department of Pharmacology, School of Medicine, UCSD, San Diego, California, USA. ${ }^{17}$ Neurobiology of Addiction Section, Intramural Research Program, National Institute on Drug Abuse, Baltimore, Maryland, USA.

Chronic alcohol abuse has a detrimental effect on the brain and liver. There is no effective treatment for these patients, and the mechanism underlying alcohol addiction and consequent alcoholinduced damage of the liver/brain axis remains unresolved. We compared experimental models of alcoholic liver disease (ALD) and alcohol dependence in mice and demonstrated that genetic ablation of IL-17 receptor A (IL-17 $\mathrm{ra}^{-/-}$) or pharmacological blockade of IL-17 signaling effectively suppressed the increased voluntary alcohol drinking in alcohol-dependent mice and blocked alcohol-induced hepatocellular and neurological damage. The level of circulating IL-17A positively correlated with the alcohol use in excessive drinkers and was further increased in patients with ALD as compared with healthy individuals. Our data suggest that IL-17A is a common mediator of excessive alcohol consumption and alcohol-induced liver/brain injury, and targeting IL-17A may provide a novel strategy for treatment of alcohol-induced pathology.

Authorship note: HYM and XL contributed equally to this study.

Conflict of interest: The authors have declared that no conflict of interest exists.

Copyright: (c) 2020, American Society for Clinical Investigation.

Submitted: July 10, 2019 Accepted: December 26, 2019 Published: February 13, 2020.

Reference information: JCI Insight. 2020;5(3):e131277. https://doi.org/10.1172/jci. insight.131277.

\section{Introduction}

Alcohol use disorder is a chronic, relapsing disorder manifested by repetitive and compulsive consumption of alcohol, followed by a dysregulated emotional state during abstinence (1). The central nervous system (CNS) is a major target of chronic alcohol toxicity, which can lead to a range of complications with progressive neurocognitive disorders and brain pathologies, characterized by neuroinflammation, neuronal apoptosis, and reactive astrogliosis (2). Patients addicted to alcohol often develop alcoholic liver disease (ALD), which progresses from steatosis to steatohepatitis and cirrhosis (3). Loss of detoxifying functions in the alcohol-damaged liver results in release of hepatotoxins and microbial metabolites into circulation and development of systemic inflammation (4) that further increases permeability of the blood-brain barrier (BBB), facilitates alcohol-induced brain injury (5), and as a result, may perpetuate and promote the addictive behavior. However, no common underlying mechanism for alcohol-induced liver/brain injury and alcohol addiction has been identified. 
Table 1. Histopathological comparison of livers from mice with acute or chronic ASH, or IG alcohol feeding-induced liver fibrosis

\begin{tabular}{|c|c|c|c|}
\hline ALD stages & Acute ASH & Chronic ASH & Alcoholic liver fibrosis \\
\hline Serum alcohol, mg/dL & $370 \pm 10$ & $437 \pm 23$ & $471 \pm 12$ \\
\hline \multicolumn{4}{|l|}{ Biochemical parameters } \\
\hline Serum ALT (IU/L) & $110 \pm 32$ & $121 \pm 30$ & $215 \pm 51$ \\
\hline Live triglycerides (nmol/gL) & $73 \pm 5.4$ & $94 \pm 10.3$ & $151 \pm 11.2$ \\
\hline Steatosis \% & 0 & $28 \pm 7$ & $75 \pm 7$ \\
\hline Lobular inflammation grade & 1 & $1-2$ & $1-3$ \\
\hline Portal inflammation grade & 0 & 0 & $0-2$ \\
\hline Fibrosis stage & 0 & $0-1$ & $2-3$ \\
\hline \multicolumn{4}{|l|}{ Histological parameters } \\
\hline
\end{tabular}

WT mice (male C57BL/6, 8 weeks old, $n=7-10 /$ group, 2-3 independent experiments per model of alcohol-induced liver injury) were subjected to acute ASH, chronic ASH, and IG alcohol feeding-induced liver fibrosis and compared with pair-fed (control) mice ( $n=3-6 /$ model). Hepatic liver injury was graded by a pathologist based on histopathology ( $\mathrm{H} \& \mathrm{E}$, Sirius red, and anti-F4/80+ staining); biochemical parameters were analyzed (vs. corresponding pair-fed control mice). One-way ordinary ANOVA for multiple comparisons was applied.

IL-17A is produced mainly by CD4+ Th17 cells and signals through the ubiquitously expressed receptor IL-17RA (6). Expression of IL-17RA is upregulated in IL-17-responsive tissues, including liver (7) and brain (8). Differentiation of Th0 naive cells into Th17 cells is triggered by TGF- $\beta 1$ and IL-6 (9) (the cytokines that also drive development of liver fibrosis; ref. 10) and requires activation of transcription factor retinoic acid receptorrelated orphan nuclear receptor $\gamma \mathrm{t}$ (ROR $\gamma \mathrm{t}$, which regulates the development of Th17 cells, $\gamma \delta \mathrm{T}$ cells, and other innate lymphoid cells that express Th17 cell-like cytokines; refs. 6, 11). Th17 cells mediate immune responses against extracellular bacteria and fungi, and their dysregulation is linked to autoimmune diseases, such as asthma, rheumatoid arthritis, and psoriasis (11). IL-17 signaling has been implicated in the pathogenesis of lung, liver, and skin fibrosis $(7,12,13)$. Recent studies have linked IL-17A to brain disorders, such as autism-related symptoms (14), morphine withdrawal syndrome (15), and development of neuroinflammation and anxiety-related behavior in alcohol-fed mice (16), suggesting that IL-17 signaling may provide a link between excessive alcohol drinking that combines alcohol addiction and alcohol-induced liver and brain pathology.

Although the role of IL-17 signaling in the pathogenesis of chronic alcohol abuse has not been investigated, several lines of evidence suggest IL-17A may be one of the missing common mediators (5). Patients with ALD often exhibit elevated IL-17A, which correlates with the severity of alcoholic steatohepatitis and fibrosis (17). Increased numbers of circulating Th17 cells were reported in active drinkers but not in abstinent alcoholics (18).

Alcoholism and ALD represent complex interactions of the brain/liver axis. There is not a single model that can recapitulate development of alcohol dependence and progression of ALD in mice. Here we used 4 experimental models of ALD progression and alcohol dependence in mice to investigate the role of IL-17 signaling in excessive alcohol drinking and to explore the novel concept that IL-17 is the cytokine that is the common mediator. Chronic high doses of alcohol raised the levels of IL-17A in mouse liver, reproducing the findings in patients. Genetic and pharmacological inhibition of IL-17A revealed that IL-17 signaling facilitates progression of ALD, alcohol-induced brain injury, and escalation of voluntary alcohol drinking in mice.

\section{Results}

Activation of IL-17 signaling is associated with ALD progression in mice. WT mice were subjected to 3 experimental models of alcohol-induced liver injury: (a) acute alcoholic steatohepatitis (ASH) (10 days, ethanol Lieber-DeCarli diet + single alcohol binge) (19), (b) chronic ASH (8 weeks, ethanol Lieber-DeCarli diet + weekly binges) (20), and (c) liver fibrosis in intragastric (IG) alcohol-fed mice ( 8 weeks, ad libitum high-cholesterol/saturated fat diet + alcohol infusion + binges) (21). Alcohol-induced liver injury was assessed by grading hepatic steatosis, 


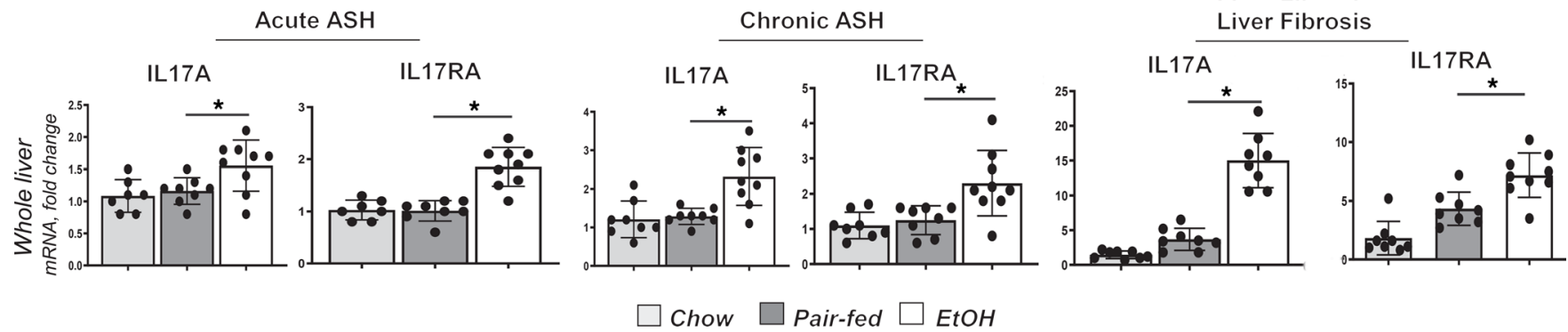

Figure 1. Progression of ALD is associated with activation of IL-17 signaling. Livers from WT mice subjected to acute ASH, chronic ASH, or IC alcohol feeding-induced liver fibrosis (male C57BL/6, 8 weeks old, $n=7-10 / g r o u p, ~ 2-3$ independent experiments/model) were analyzed by quantitative real-time PCR. Data are fold induction versus chow-fed mice (compared with the appropriate pair-fed control mice). One-way ordinary ANOVA for multiple comparisons was applied. ${ }^{*} P<0.05$ (see Supplemental Figures 1 and 2 ). EtOH, ethanol.

inflammation, and fibrosis (Table 1, Supplemental Figures 1-3, and Supplemental Text 1; supplemental material available online with this article; https://doi.org/10.1172/jci.insight.131277DS1). These models closely recapitulated the stages of ALD in patients and, therefore, were used in this study to investigate the role of IL-17 signaling in ALD progression. Hepatic expression of IL-17A and IL-17RA mRNA were progressively increased in mice with acute (2-fold) or chronic (3-fold) ASH and alcohol-induced liver fibrosis (3- to 6-fold), compared with the corresponding pair-fed controls (Figure 1), suggesting that activation of IL-17 signaling correlates with the severity of alcohol-induced liver injury in mice.

Development of acute ASH is similar in WT and IL-17ra- mice. The contribution of IL-17 signaling to ALD progression was further investigated using WT and IL-17ra ${ }^{-/-}$mice. Both WT and IL-17 $\mathrm{ra}^{-1-}$ mice developed similar characteristics of acute ASH and liver injury (alanine aminotransferase [ALT] $110 \pm 40 \mathrm{IU} / \mathrm{L}$ vs. $104 \pm 36 \mathrm{IU} / \mathrm{L}$; Supplemental Figure 1, A and B, and Supplemental Texts 2 and 3), suggesting that IL-17A does not significantly affect the onset of ALD in mice.

Development of chronic ASH is attenuated in IL-17ra-1- mice. When development of chronic ASH was compared in WT and IL-17 $\mathrm{ra}^{-/-}$mice, hepatocellular injury, lipid peroxidation, and expression of cytochrome P450 family 2 subfamily E member 1 and NADPH oxidase 4 (NOX4) were markedly reduced in IL-17 $\mathrm{ra}^{-/-}$mice (Supplemental Figure 2, A-F) and correlated with downregulation (about 2-fold) of hepatic IL-8, macrophage inflammatory protein 1, and IL-6 and upregulation of IL-10 mRNA (3-fold, Supplemental Figure 2, E-I), suggesting that IL-17 signaling facilitates hepatocellular damage and negatively regulates IL-10 production.

IL-17ra- mice are protected from alcohol-induced liver fibrosis. WT mice (male C57BL/6, 12 weeks old) were subjected to the model of alcohol-induced liver fibrosis using IG alcohol feeding (compared with the pair-fed mice) (21). The causal contribution of IL-17 signaling to the experimental model of alcohol-induced liver fibrosis was investigated using WT and IL-17ra ${ }^{-/-}$mice. Liver injury, hepatic steatosis, triglyceride synthesis, lipid peroxidation (6-fold), and oxidative stress ( 2 NOX1/2 fold) were strongly suppressed in IG alcohol-fed IL-17ra ${ }^{-/}$mice (vs. WT mice; Figure 2A, Supplemental Figure 3, A-F, and Supplemental Text 4). (Of note, although hepatic steatosis was markedly reduced in IL-17RA ${ }^{-/-}$mice, expression of sterol regulatory element-binding protein 1c, PPAR $\alpha$, and PPAR $\gamma$ mRNA was not affected by IL-17RA deficiency, suggesting that IL-17 signaling regulates hepatic lipogenesis by a mechanism distinct from de novo lipogenesis). Furthermore, IL-17 $\mathrm{ra}^{-/-}$mice were protected from alcohol-induced liver fibrosis, as shown by reduction of the fibrous scar (3-fold Sirius red-stained area) and downregulation of fibrogenic (Colla1, $\alpha$-SMA, Timp1) and inflammatory gene expression (I16, I11B, TNFA; Figure 2, B-D, and Supplemental Figure $3 \mathrm{G}$ ). Overall, IL-17 signaling appears to regulate progression of ASH to fibrosis, and inhibition of IL-17A thus may be a potential target for patients with advanced stages of ALD. TCR $\beta^{+} \mathrm{CD} 4^{+} \mathrm{T}$ cells were identified as a major source of IL-17A in fibrotic livers (Supplemental Figure 3I).

Blockade of IL-17 signaling ameliorates alcohol-induced liver fibrosis. The effect of IL-17A blockade on development of alcohol-induced liver fibrosis was tested in IG alcohol-fed WT mice therapeutically (10 days after induction of liver injury) treated with neutralizing anti-IL-17A Ab (22) (which blocks IL-17A protein vs. IgG) or ROR $\gamma$ t inhibitor (VPR-254 from Visionary Pharmaceuticals), which prevents differentiation of Th17 cells, versus vehicle (Figure 3, Supplemental Figure 4A, and ref. 23). 
A

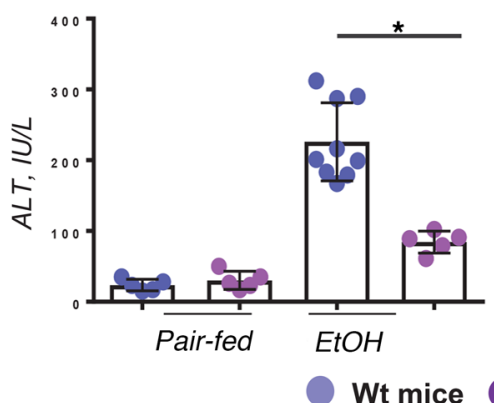

Wt mice

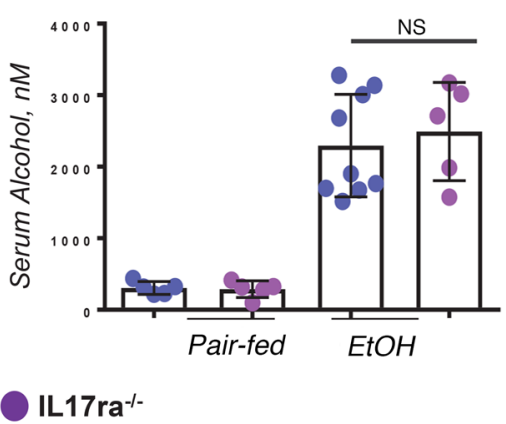

$\alpha$ SMA

C

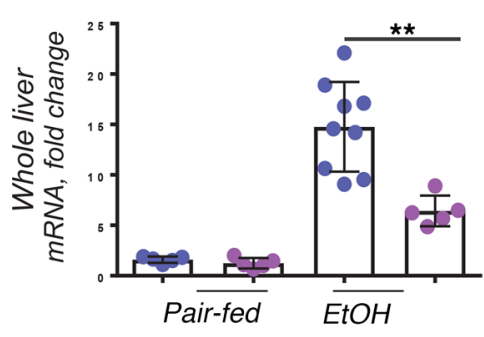

D

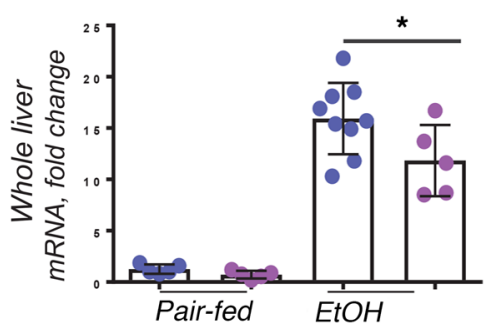

IL-6

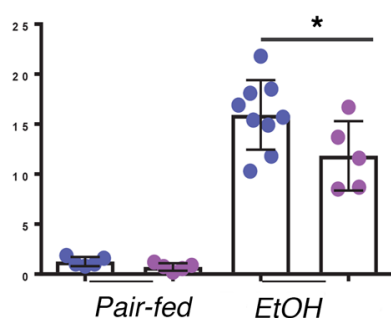

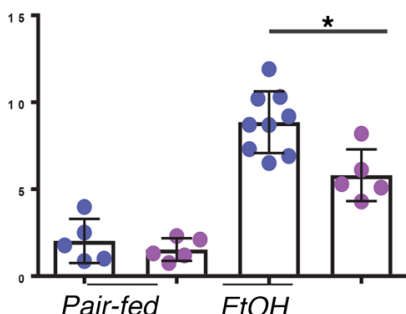

Pair-fed

$\mathrm{EtOH}$

IL-1 $\beta$

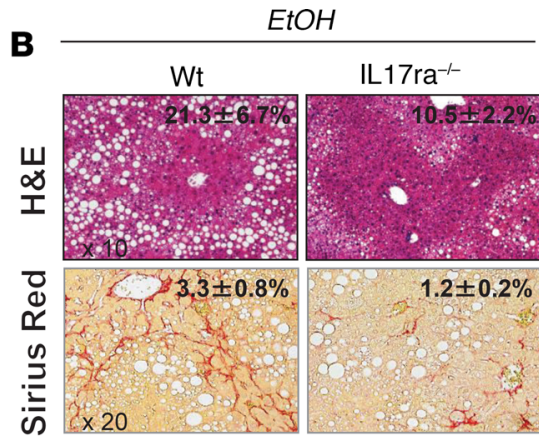

TNF- $\alpha$

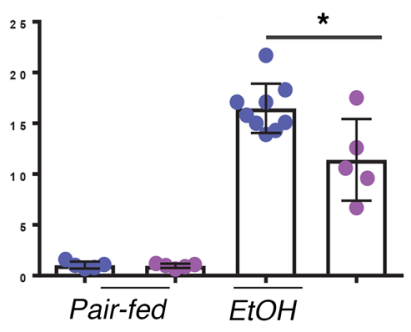

TIMP1

TGF- $\beta 1$
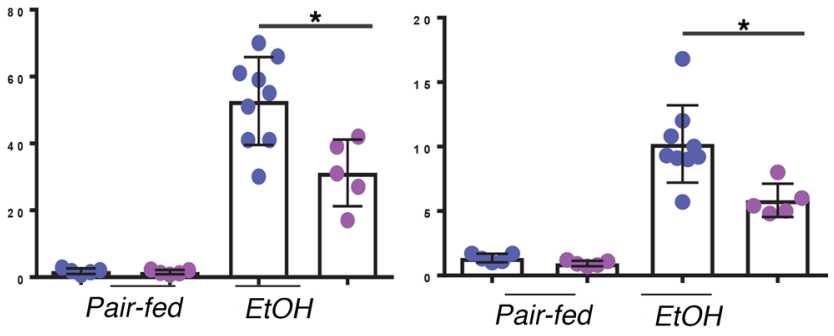

Figure 2. IL-17ra-/- mice are protected from alcoholic liver fibrosis. WT and IL-17ra-/- littermates (male $[57 \mathrm{BL} / 6,12 \mathrm{weeks}$ old) were pair-fed ( $n=5-7 / g r o u p)$ or IC alcohol fed ( $n=7-10 /$ group, 2 independent experiments). (A) Serum levels of ALT (IU/L) and EtOH (nM) were measured. (B) Livers were stained with $\mathrm{H} \& \mathrm{E}$ and Sirius Red, and positive area was calculated as percentage; micrographs are shown using $\times 20$ objective. Expression of (C) fibrogenic and (D) inflammatory gene mRNA. Data are shown as fold change (vs. IG alcohol-fed WT mice). One-way ordinary ANOVA for multiple comparisons was applied. ${ }^{*} P<0.05$; ${ }^{* *} P<0.01$ (see Supplemental Figure 3). Col1 $\alpha 1$, collagen type $1 \alpha 1 ; \alpha$-SMA, $\alpha$-smooth muscle actin; TIMP1, tissue inhibitor of metalloproteinase 1.

Administration of anti-IL-17A Ab to IG alcohol-fed mice reduced serum levels of IL-17A protein (1.4 fold) and other inflammatory cytokines (Figure 3A and Supplemental Figure 4, B and D), improved liver function (Figure 3B), reduced hepatic steatosis (2-fold, Figure 3C), and decreased alcoholic liver fibrosis by approximately $50 \%$, as shown by reduced Sirius red-stained area (2-fold) and downregulation of fibrogenic and inflammatory genes (vs. IgG-treated mice; Figure 3, C-E, and Supplemental Figure 4, E-G). Gene expression profiling revealed that depletion of circulating IL-17A prevented hepatotoxicity and lipogenesis in alcohol-damaged hepatocytes (Supplemental Figure 5, A-C), and suppressed activation of Kupffer cells (Supplemental Figure 5, D and E). A similar effect was observed in IG alcohol-fed WT mice treated with ROR $\gamma$ t inhibitor. Taken together, we demonstrate that blockade of IL-17 signaling can block alcohol-induced liver fibrosis to a level similar to that observed in IL-17 $\mathrm{ra}^{-/-}$mice.

$I L-17 \mathrm{ra}^{-/-}$mice are protected from alcohol-induced dysbiosis. Alcohol-induced dysbiosis has been implicated in the pathogenesis of ALD (24-26) and also contributes to activation of Th17 cells (27). Here we demonstrate that IL-17 signaling critically affects alcohol-induced changes in the intestinal microbiota. IG alcohol-fed IL-17 $\mathrm{ra}^{-/-}$mice were largely protected from alcohol-induced dysbiosis versus WT littermates (Supplemental Figure 6A). However, blockade of IL-17A suppressed alcohol-induced fibrosis but did not reverse alcohol-induced dysbiosis in IG alcohol-fed mice (Supplemental Figure 6B), suggesting that 
A

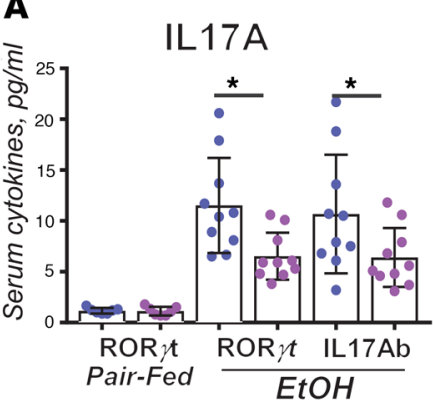

C

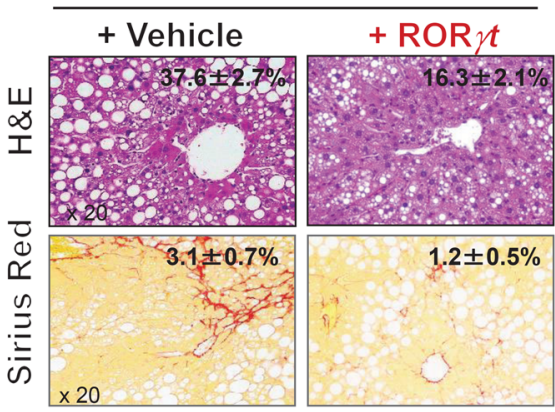

D
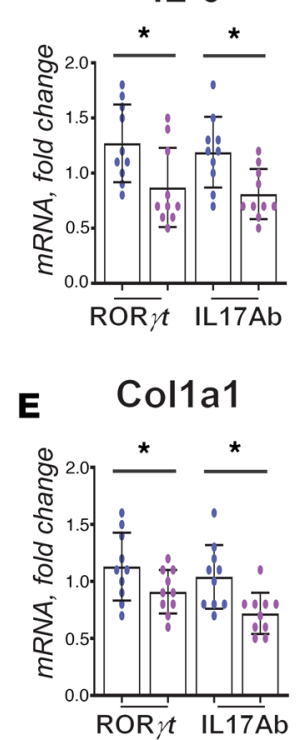

B

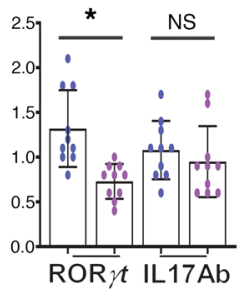

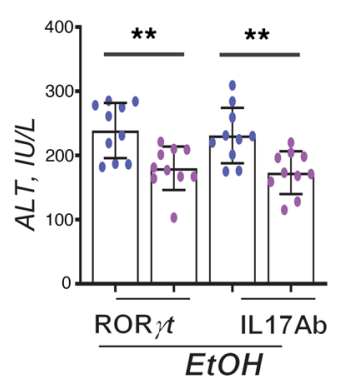

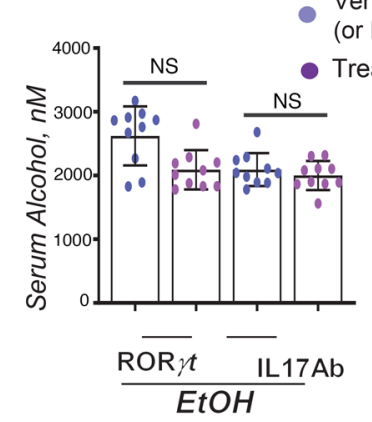

$\mathrm{EtOH}$
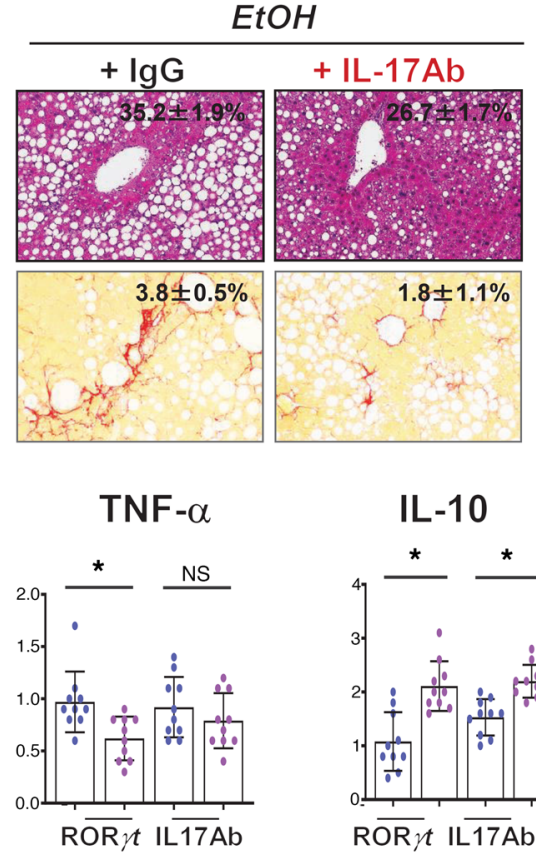

TNF- $\alpha$

TIMP1

IL-10

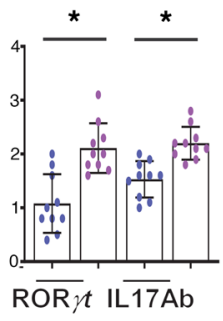

TGF- $\beta 1$

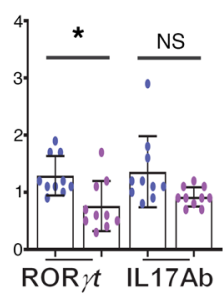

Figure 3. Blockade of IL-17A reduces alcoholic liver fibrosis by approximately $\mathbf{5 0 \%}$. IG alcohol-fed WT mice (male C57BL/6, 12 weeks old, $n=10-12$ / group, 3 independent experiments) were treated with ROR $\gamma \mathrm{t}$ inhibitor (50 $\mathrm{mg} / \mathrm{kg} /$ weight vs. vehicle, oral gavage 4 times per week, or vehicle) or antiIL-17A Ab $(25 \mathrm{mg} / \mathrm{kg} /$ weight vs. IgG, i.p., 1 time per week). (A) Serum levels of IL-17A (pg/mL), (B) ALT (IU/L), and blood EtOH (nM) were measured. (C) Livers were stained with $\mathrm{H} \& \mathrm{E}$ and Sirius Red, positive area was calculated as percentage, and micrographs are shown using $\times 20$ objective. (D) Expression of fibrogenic and $(\mathbf{E})$ inflammatory gene mRNA. Data are shown as fold change (vs. IgG-treated mice). One-way ordinary ANOVA for multiple comparisons was applied. ${ }^{*} P<0.05 ;{ }^{* *} P<0.01$ (see Supplemental Figures 4-6).

IL-17A plays a role in the development of alcohol-induced dysbiosis, but once engaged, alcohol-induced dysbiosis is refractory to IL-17A blockade. Results also suggest that recovery from ALD might not depend on microbiome restoration.

IL-17A facilitates activation of primary astrocytes and microglia. Alcohol-induced liver and brain injury are interconnected $(28,29)$. Hepatic stellate cells (HSCs) in the liver exhibit similarities with brain astrocytes, while Kupffer cells and microglia both function as macrophages of their respective organs (30-32). Because IL-17A targets HSCs and Kupffer cells in the injured liver (7), we tested whether IL-17A can also activate astrocytes and microglia in the brain by examining the effect of IL-17A on immunoregulatory properties of cultured astrocytes and microglia (33). In response to IL-17A stimulation, $\mathrm{GFAP}^{+}$astrocytes and $\mathrm{GFAP}^{-} \mathrm{F} 4 / 80^{+}$microglia upregulated expression of IL-17RA, indicating that both cell types respond to IL-17 signaling (Supplemental Figure 7A and Supplemental Text 5). IL-17A induced activation and proliferation in astrocytes (fold upregulation by 1.8 GFAP and by 1.5 cyclin D1 [CCND1]) and stimulated microglia cells to produce TGF- $\beta 1$ (1.3-fold), TNF- $\alpha$ (1.7-fold), 
and IL-1 $\beta$ (1.6-fold). In turn, TGF- $\beta 1$ and IL-1 $\beta$ exerted strong inflammatory responses in cultured astrocytes (Supplemental Figure 7, B-D). We concluded that IL-17A regulates astrocyte activation, either directly (facilitating astrocyte proliferation) or indirectly (via activation of microglial cells).

Systemic IL-17A blockade ameliorates astrogliosis in the brains of IG alcohol-fed mice. The effect of systemic IL-17A blockade on alcohol-induced brain injury was evaluated in IG alcohol-fed mice. After 10 days of alcohol feeding, administration of anti-IL-17A Ab (or ROR $\gamma t$ inhibitor) significantly suppressed activation of $\mathrm{Iba}^{+}$microglial cells (Figure 4, A-C), especially in the hippocampus, as demonstrated by reduced cellular size $\left(1.1 \pm 0.4 \mu \mathrm{m}^{2}\right.$ per cell vs. $2.4 \pm 0.3$ control mice; Supplemental Figure $7, \mathrm{~A}$ and $\mathrm{B}$ ) and downregulation of Iba1 (1.35-fold), TNF- $\alpha$, and IL-1 $\beta$ mRNA in the whole brains of IG alcohol-fed WT mice (Figure 4, D and E, and Supplemental Figure 8C). Neuronal apoptosis was assessed by immunostaining for active caspase-3. Expression of active caspase-3 was observed only in the cerebellum of IG alcohol-fed mice and was significantly reduced (2.5-fold) mice treated with anti-IL-17A Ab $(2.0 \pm 0.8 \%$ positive area vs. $5.1 \pm 1.0 \%$ in IgG-treated mice; Supplemental Figure $8 \mathrm{D})$. Furthermore, blockade of IL-17A prevented astrocyte proliferation and activation (astrogliosis; ref. 34; Figure 4, A and B), as demonstrated by decreased astrocyte size $\left(1.1 \pm 0.4 \mu \mathrm{m}^{2}\right.$ per cell vs. 2.4 $\pm 0.3 \mu \mathrm{m}^{2}$ in IgG-treated mice) and reduced expression of GFAP mRNA in the whole brain (2-fold) and in specific brain structures (1.5-fold frontal cortex; 1.7-fold cerebellum; and 3-fold hippocampus; Supplemental Figure 8E). Taken together, inhibition of IL-17A overall ameliorated alcohol-induced brain injury and suppressed alcohol-induced astrogliosis in IG alcohol-fed mice.

Development of astrogliosis is associated with neuroinflammation in IG alcohol-fed mice. Many cell types respond to IL-17A stimulation by upregulation of IL-17RA (ref. 6 and Supplemental Figure 9A). Here we demonstrate that IL-17RA mRNA was induced in the cortex (1.3-fold), hippocampus (1.7-fold), and cerebellum of IG alcohol-fed mice (1.4-fold vs. chow-fed mice, Supplemental Figure 9B), suggesting that chronic exposure to alcohol activates IL-17 signaling in the CNS. Although brain cells did not express IL-17A mRNA (Supplemental Figure 9C), IL-17A protein was detected in the CSF of IG alcohol-fed mice (6-fold vs. pair-fed mice), suggesting that IL-17A enters the CSF from circulation (Figure 4D). The CSF levels of IL-1 $\beta$, TGF- $\beta 1$, and TNF- $\alpha$ cytokines were also elevated and correlated with increased mRNA expression of these cytokines in the brains of IG alcohol-fed mice (Figure 4D and Supplemental Figure 9D), indicating that brain cells serve as a significant source of cytokines and contribute to neuroinflammation. Administration of anti-IL-17A Ab suppressed expression of TNF- $\alpha$ in the brains of IG alcohol-fed mice and blocked alcohol-induced neuropathology (Figure 4E).

$I L-17 A$ passes across the damaged BBB of IG alcohol-fed WT mice. To determine the mechanism by which IL-17A enters the CSF, the integrity of the BBB was examined in IG alcohol-fed mice. An increase of albumin content (3-fold, which was indicative of increased BBB permeability) was measured in the CSF of IG alcohol-fed WT mice (vs. pair-fed mice, Supplemental Figure 10A). Immunostaining for tight junction protein Zo-1 (to visualize blood vessels) and albumin revealed the presence of extravasated albumin in the brain parenchyma of IG alcohol-fed mice (but not in pair-fed mice, Supplemental Figure 10B and white arrows in Supplemental Figure 10C). We concluded that serum IL-17A enters the brain across the damaged BBB. Our data are in concordance with previous reports demonstrating that the integrity of the BBB is compromised by chronic alcohol consumption in patients and contributes to neuroinflammation (35). IL-17A blockade did not ameliorate BBB disruption in IG alcohol-fed mice (Supplemental Figure 10, A-C), indicating that IL-17 signaling might not be directly responsible for the loss of BBB integrity in these mice.

Serum levels of IL-17A are elevated in patients with severe alcohol use disorder. Serum levels of IL-17A were measured in healthy individuals and excessive drinkers with a history of ALD and without history of alcohol-induced liver injury (Figure 5A). High levels of serum IL-17A were detected in excessive drinkers as compared with healthy individuals. The correlation between excessive alcohol consumption and serum levels of IL-17A was established in patients with normal liver function (ALT < $40 \mathrm{U} / \mathrm{L}$ ) or abnormal ALT ( $\geq 40$ U/L; Supplemental Tables 1 and 2). Strikingly, the level of circulating IL-17A was strongly increased (10-fold) in excessive drinkers with normal liver function (ALT ranging 25-67 U/L) and was further elevated in excessive drinkers with ALD (16-fold vs. healthy individuals), suggesting that upregulation of serum IL-17A might be associated with development of alcohol use disorder in excessive drinkers.

Circulating IL-17A is upregulated in alcohol-dependent mice. The potential role of IL-17 signaling in the motivational aspects of chronic alcohol abuse was studied using a mouse model of ethanol dependence that combines chronic intermittent inhalation of ethanol vapor (CIE, repeated rounds of intoxication: 4 cycles, 16 hours, 
A

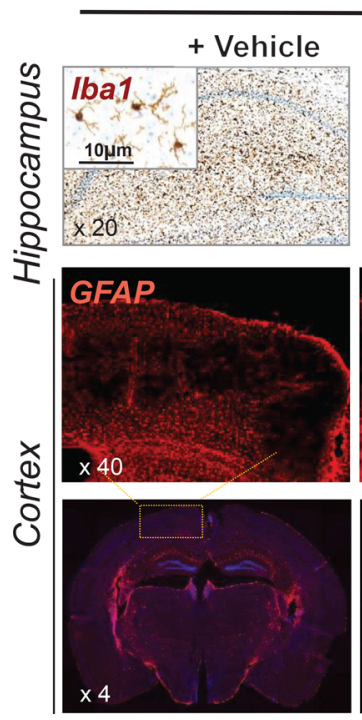

$+\operatorname{ROR} \gamma t$
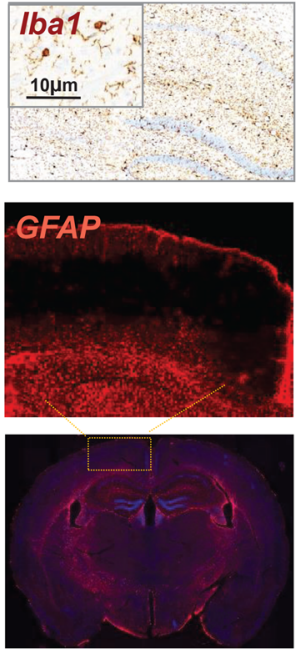

$\mathrm{EtOH}$
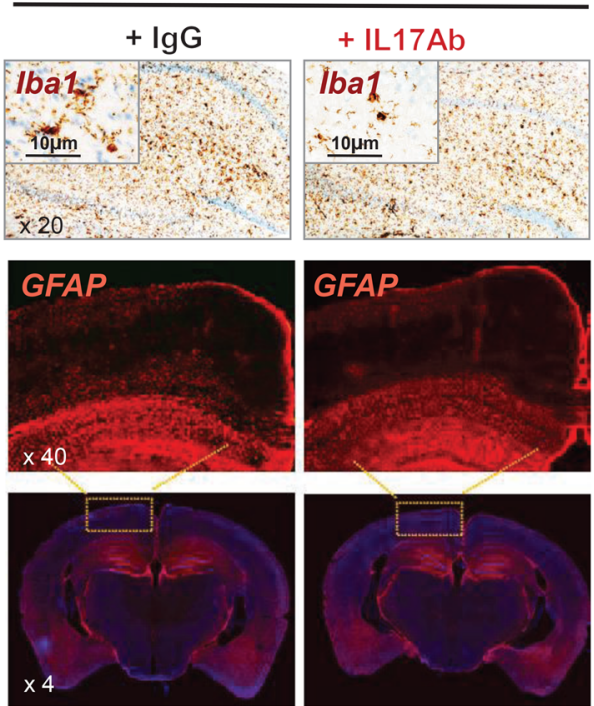

\section{GFAP}
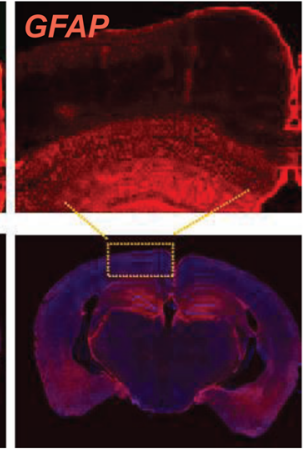

B

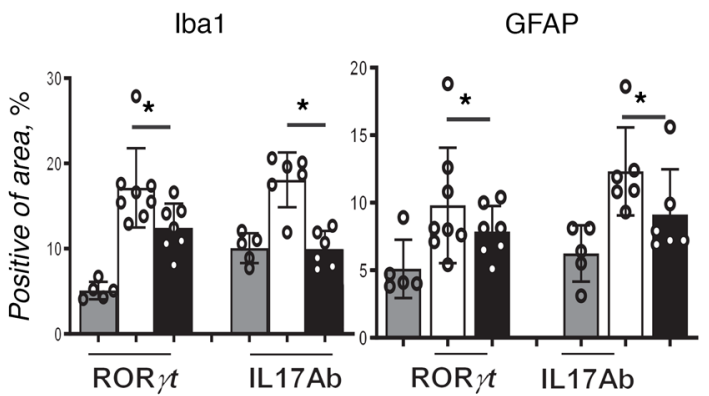

C

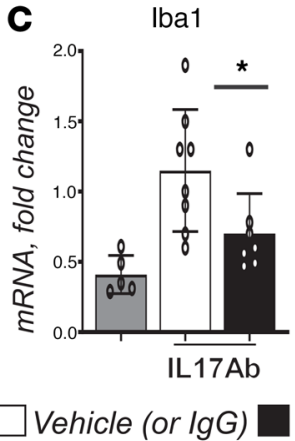

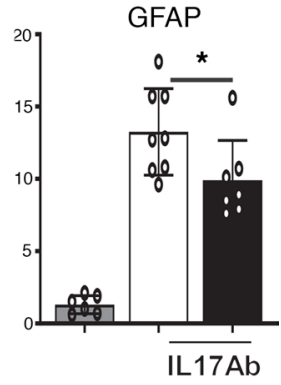

Figure 4. Blockade of IL-17A ameliorates alcohol-induced brain injury and neuroinflammation. Brains and cerebrospinal fluid (CSF) were collected from IG alcohol-fed WT mice with or without $\mathrm{ROR} \gamma \mathrm{t}$ inhibitor or with or without anti-IL-17A Ab (male C57BL/6, 12 weeks old, $n=12$ /group, 3 independent experiments). (A) Coronal brain sections were immunostained for induction of brown adipocytes 1 (Iba1) (hippocampus, $\times 20$ and $\times 100$ objectives) or glial fibrillary acidic protein (GFAP) (cortex, $x 4$ and $\times 40$ objectives). (B) Positive area was calculated as percentage. (C) Expression of Iba1 and GFAP mRNA. Data are shown as fold change. (D) CSF levels of IL-17A and TNF- $\alpha$ were measured by ELISA (pg/mL). (E) Expression of TNF- $\alpha$ mRNA was measured. Two-tailed Student's $t$ test and 1-way ordinary ANOVA for multiple comparisons were applied. ${ }^{*} P<0.05$ (see Supplemental Figures 7-10).

D

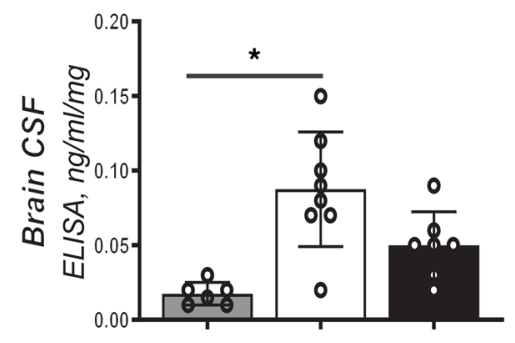

E

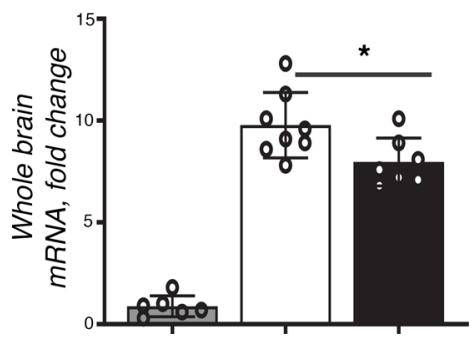

Wt mice:

Pair-fed

$\mathrm{EtOH}+\lg G$

$E t O H+I L 1$

target blood alcohol level $=200 \mathrm{mg} / \mathrm{dL}$, followed by 8 hours' withdrawal) with voluntary ethanol drinking during limited-access 2-bottle choice sessions (2BC, water vs. ethanol 15\% v/v, $2 \mathrm{~h} / \mathrm{d}, 3-7$ days' withdrawal from vapor; Figure 5B). In this model, CIE-exposed mice became alcohol dependent and developed a gradual escalation of voluntary ethanol drinking compared with nondependent (Air) mice (36). Development of alcohol dependence in CIE mice was also associated with a significant increase of serum IL-17A (1.3-fold, vs. Air mice) that persisted for more than 4 days into withdrawal from ethanol vapor (Figure 5, B and C, and Supplemental Figure 11A). Serum levels of other inflammatory cytokines (IL-1 $\beta$ and TNF- $\alpha$ ) were not changed between CIE and Air mice and were comparable to those observed in naive mice (Supplemental Figure 11A). Among all tested cytokines, IL-17A was the only cytokine upregulated in the blood of CIE mice.

Blockade of IL-17A suppressed voluntary ethanol drinking in dependent mice. On average, CIE mice consumed 3 times more alcohol than Air mice, as shown during a 2BC session at day 3 into withdrawal from ethanol vapor (Figure 6, A and B). Dependent (CIE) and nondependent (Air) mice were further divided into 2 groups $(n=$ 6/group) and treated with a single dose of anti-IL-17A Ab (25 mg/kg, i.p., or IgG). Eighteen hours later the 

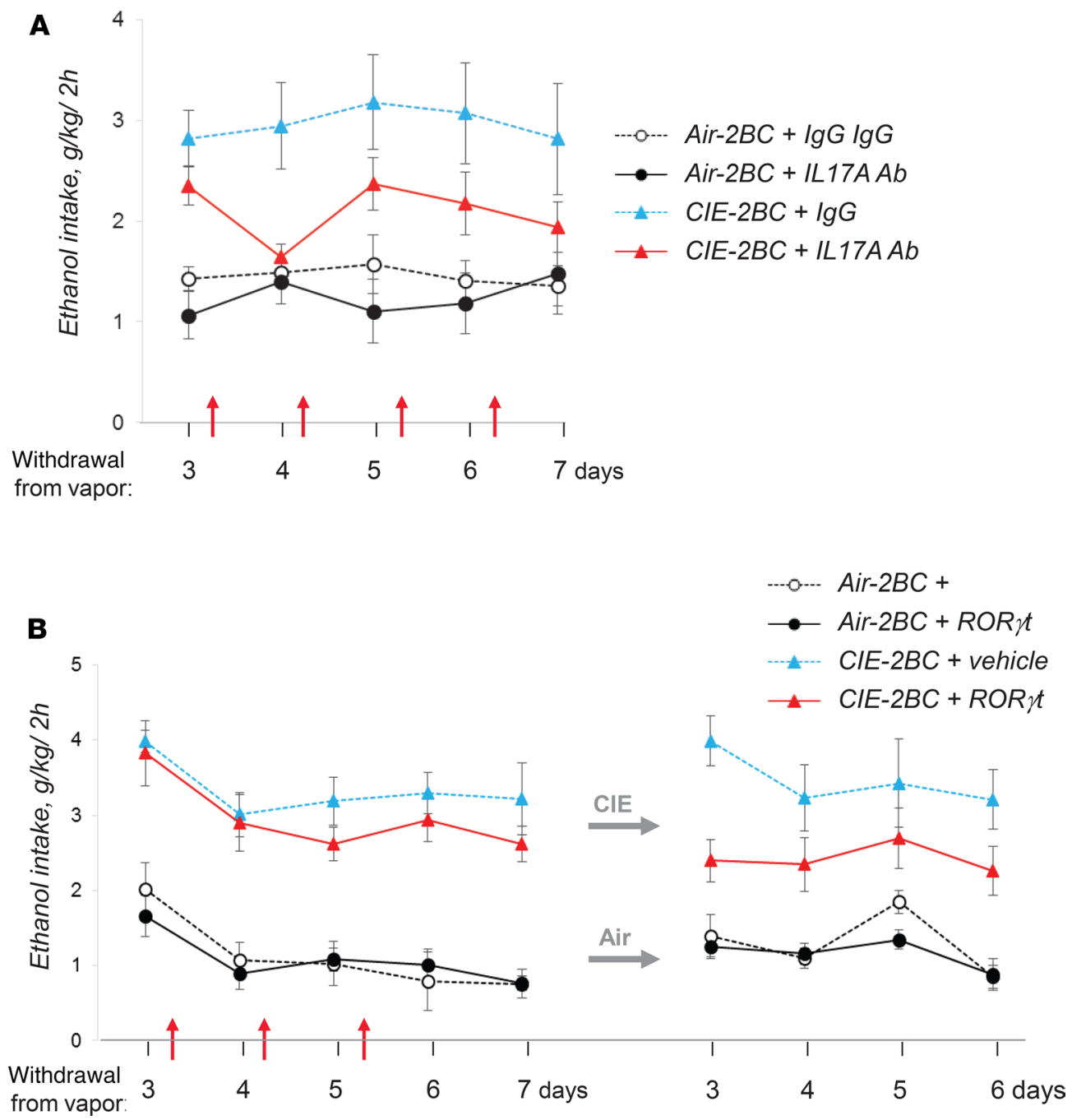

Figure 6. Inhibition of IL-17A suppresses voluntary alcohol drinking in alcohol-dependent mice. (A and B) Voluntary alcohol intake (g ethanol/kg body weight) was measured during 2BC sessions (2 hours) in Air and CIE mice with or without anti-IL-17A Ab (or with or without ROR $\gamma \mathrm{t}$ inhibitor). (A) Multiple doses of anti-IL-17A Ab were administered on consecutive days (the regimen is shown, red arrows). The most significant effect was detected on the first day of treatment (withdrawal day 4: effect of $\mathrm{Ab}, \mathrm{F}[1,19]=6.6$, and $P<0.05$; effect of $\mathrm{CIE}, \mathrm{F}[1,19]=9.7$, and $P<0.01$; $\mathrm{Ab}$ by $\mathrm{CIE}$ interaction, $\mathrm{F}[1,19]=5.1$, and $P<0.05$ ) and persisted throughout the week (withdrawal days 4-7: effect of $A b, F[1,19]=3.5$, and $P<$ 0.08 ; see Supplemental Figure 10). (B) Air and CIE mice with or without ROR $\gamma$ t inhibitor. There was no immediate effect of ROR $\gamma$ t inhibitor on voluntary ethanol drinking. The mice were then exposed to an additional week of CIE (or Air) and resumed 2BC sessions. The most significant effect was observed on withdrawal day 3 (effect of inhibitor, $F[1,20]=10.1$, and $P<0.01$; effect of $\mathrm{CIE}, \mathrm{F}[1,20]=47.3$, and $P<0.001$; inhibitor by $\mathrm{CIE}$ interaction, $\mathrm{F}[1,20]=7.2$, and $P<0.05$ ) but persisted throughout the week (withdrawal days 3-6: effect of inhibitor, $F[1,20]=5.5$, and $P<0.05$ ). The regimen of IL-17A inhibition is specified (red arrows). One-way ordinary ANOVA for multiple comparison was applied.

A similar effect was observed in alcohol-dependent CIE mice treated with ROR $\gamma \mathrm{t}$ inhibitor $(3 \times 50 \mathrm{mg} /$ $\mathrm{kg}$, oral gavage, 18 hours prior to $2 \mathrm{BC}$ sessions; Figure 6B). Administration of ROR $\gamma$ t inhibitor substantially reduced alcohol intake in dependent mice following exposure to an additional week of CIE, and this effect persisted for 1 week (vs. vehicle-treated dependent mice). Our data suggest that blockade of IL-17 signaling suppresses voluntary alcohol drinking in alcohol-dependent mice.

Blockade of IL-17A signaling suppresses astrocyte activation in alcohol-dependent CIE mice. To identify objective and measurable changes associated with development of alcohol dependence in mice, the brains of the alcohol-dependent and nondependent mice with or without anti-IL-17A Ab (or IgG) were collected 3 days after the last $2 \mathrm{BC}$ session and analyzed. The volume of the cortex, hippocampus, and corpus callosum were reduced in alcohol-dependent mice, (Figure 7A), while the size of GFAP ${ }^{+}$astrocytes in the hippocampus (Figure 7, B and C) and amygdala was increased (Figure 7D) and was associated with elevated levels of 
circulating IL-17A (Figure 5C). Administration of anti-IL-17A Ab reduced serum levels of IL-17A to baseline (Figure 5C) but had no effect on the overall brain volume in these mice (Figure 7A). In turn, the size of hippocampal astrocytes was reduced in CIE mice treated with anti-IL-17A Ab $\left(1.1 \pm 0.4 \mu \mathrm{m}^{2}\right.$ vs. $2.4 \pm 0.3$ $\mu^{2}$ in IgG-treated CIE mice; Figure 7E) and correlated with decreased GFAP expression in the amygdala (Figure 7E, Supplemental Figure 11, B-D, and Supplemental Figure 12). Our data indicate that depletion of circulating IL-17A reduced activation of brain astrocytes and suppressed voluntary alcohol drinking in alcohol-dependent mice (also see Supplemental Figure 12 and Supplemental Text 6).

Depletion of circulating IL-17A partially restores expression of astrocyte-regulated genes in the hippocampus of alcohol-dependent mice. Hippocampi were dissected from the brains of alcohol-dependent and nondependent mice with or without anti-IL-17A Ab and analyzed by RNA-Seq. Differential expression analysis identified 1020 alcohol dependence-specific genes (490 upregulated and 530 downregulated, $P<0.05$ ) uniquely expressed in the hippocampus of the alcohol-dependent CIE mice with IgG (vs. Air mice + IgG; the 25 most differentially expressed genes are shown, Figure 8A). Of these 1020 genes, hippocampus expression of 505 genes was reversed in CIE mice upon anti-IL-17A Ab treatment (171 were significantly reversed with $P<0.05$ ). Consistent with our findings (Figure 7, C-E), the markers of activated $\mathrm{GFAP}^{+}$astrocytes were identified among the top 490 genes uniquely induced in the hippocampus of alcohol-dependent mice (vs. Air mice; Figure 8, B and C), suggesting that reactive astrogliosis critically contributes to the pathogenesis of alcohol dependence in mice. In turn, depletion of circulating IL-17A reversed astrocyte activation (as shown by reduced expression of GFAP [ref. 37], Slc1a3 [ref. 38], Hes1/5, Notch1, Nog, Mecp2, Nr2e1, Bin1, and Atf5 markers [ref. 39]) and reinstated astrocyte-mediated pathways (ion transport, immune responses, cell adhesion, cell cycle, apoptosis, protein and lipid metabolism, and mitochondrial function; Figure 8, B and C). Anti-IL-17A Ab-treated CIE mice showed suppression of voluntary drinking (Figure 6, A and B), and this effect was associated with downregulation of genes involved in the astrocyte-neuronal regulatory networks (neurotransmitter metabolic process, regulation of neurotransmitter levels, and long-term memory pathways) and associative learning and correlated with downregulation of Junb, Fosb, CREB, Deaf1, and $\mathrm{Nfkb}$, transcription factors that drive activation of drug response genes (ref. 39; Figure 9, A and B; and Supplemental Figure 13). Together, our results suggest that IL-17 signaling regulates brain molecular targets associated with alcohol-induced astrocyte activation, especially in the hippocampus of alcohol-dependent mice, and depletion of IL-17A might be beneficial for treatment of patients with alcohol use disorder.

\section{Discussion}

We demonstrate that IL-17A is elevated in response to chronic exposure to high blood alcohol. More importantly, blockade of IL-17A either genetically or pharmacologically inhibits development of alcohol-induced liver fibrosis and suppresses excessive alcohol drinking in mice via preventing astrocytic reactivity. Thus, IL-17A is a common mediator required for many of the pathological effects of alcohol abuse.

Development of neuroinflammation and neurodegenerative disorders, neuronal death, and astrogliosis has been reported in patients with severe alcohol use disorder and has been often linked to development of liver fibrosis (35). Elevated levels of circulating and hepatic IL-17A in patients with alcohol-induced liver fibrosis have been well documented, but only recently was IL-17A linked to alcohol dependence in patients and experimental animals $(16,18,40)$. Our study provides evidence that excessive drinking is associated with a strong increase of serum IL-17A levels in patients, even while their liver function is still normal (Figure 5A), suggesting that systemic release of IL-17A precedes alcohol-induced liver injury. Serum levels of IL-17A are further increased in patients with alcohol-induced liver injury (Figure 5A) and correlate with progression of alcohol-induced liver fibrosis (7).

IL-17A signaling facilitates alcohol-induced liver injury. Similar to that observed in patients $(7,17)$, IL-17A was increased in mice with alcohol-induced liver fibrosis. Here, we demonstrate that IL-17A plays an important role in patients with ALD and ALD-like effects in mice, and we suggest that IL-17A is a significant activator of fibrosis in multiple organs and tissues $(7,12,13)$. Our data demonstrate that IL-17A regulates a variety of responses to alcohol in the damaged liver, including inflammation (IL-6, IL-1 $\beta$, TNF- $\alpha$, and ROS production) and fibrosis (TGF- 31 , activation of HSCs/myofibroblasts, Figure 2). Although IL-17 signaling has been implicated in the pathogenesis of experimental nonalcoholic steatohepatitis (NASH) (41), responses to IL-17A (steatohepatitis and fibrosis) were strongly amplified in alcohol-fed mice (Supplemental Figure 3). Thus, our results provide a mechanism for previous studies that have demonstrated that elevated levels of serum and hepatic IL-17A correlate with severity of ASH and fibrosis in patients with severe alcohol use disorder $(7,17)$. 
A

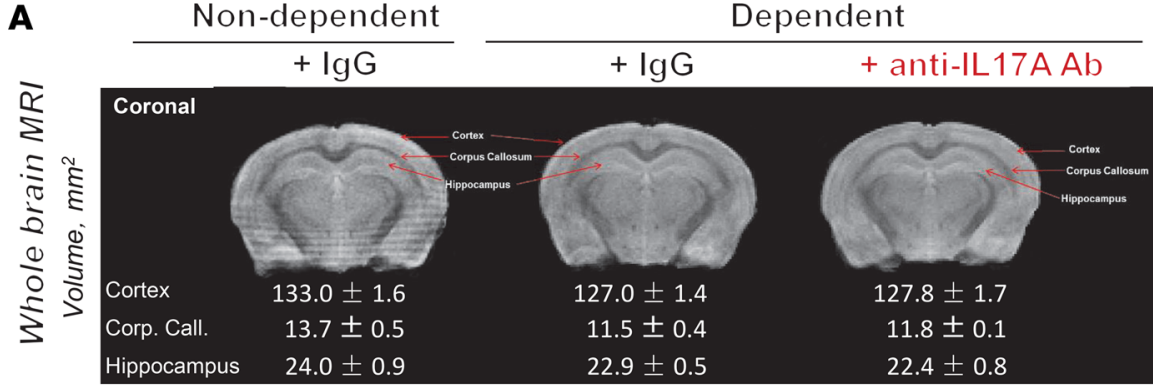

B

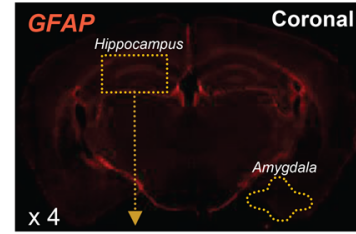

C

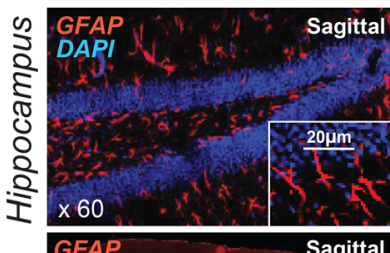

D
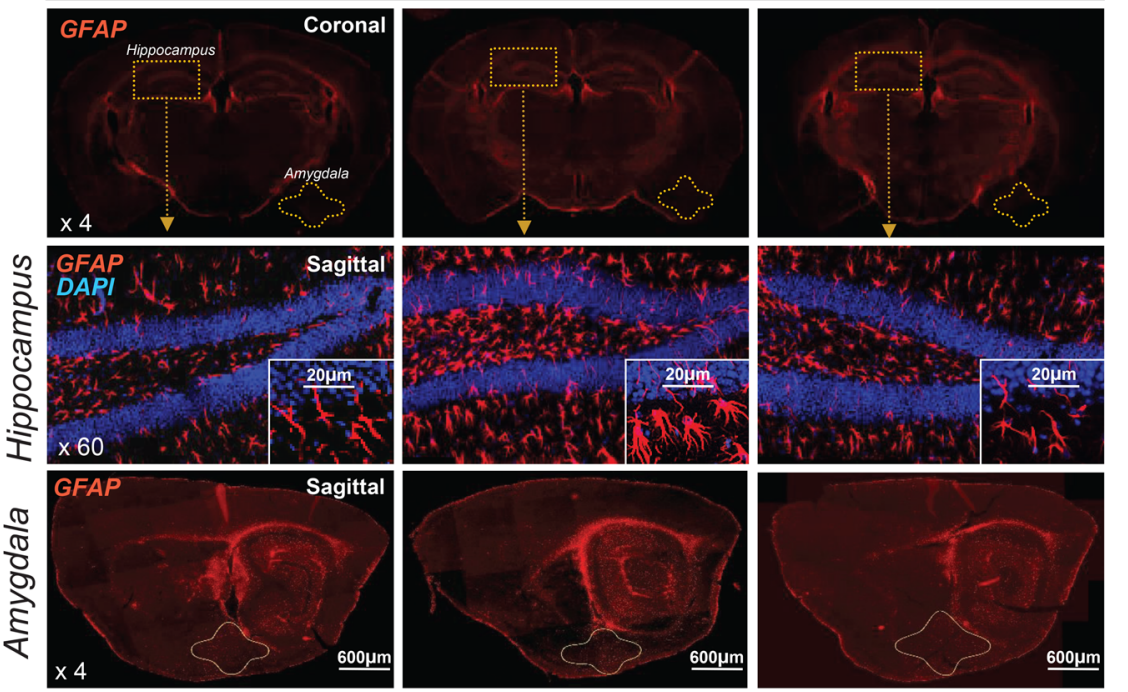

E
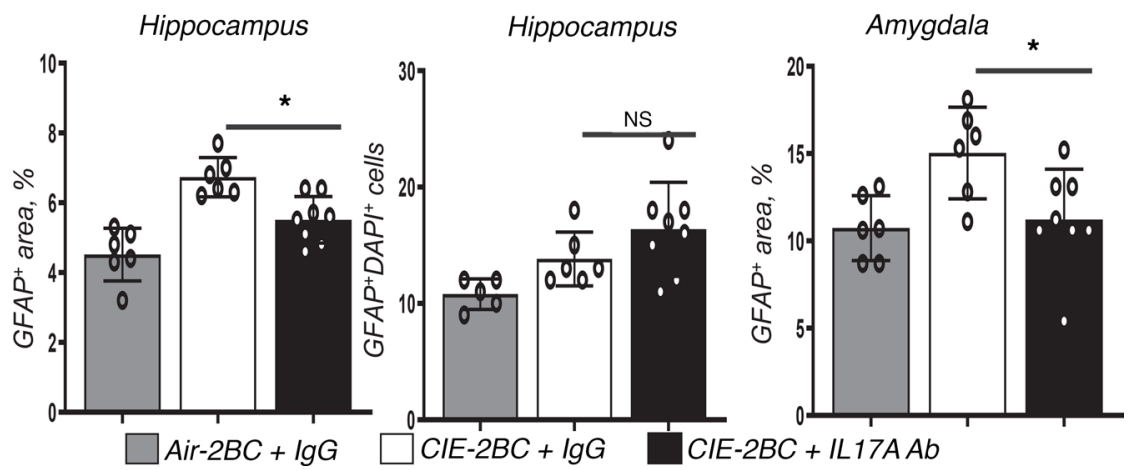

Figure 7. Inhibition of IL-17A was associated with downregulation of GFAP in the hippocampus and amygdala of alcohol-dependent mice. Brains from Air and CIE mice with or without anti-IL-17A Ab were analyzed by (A) whole-brain MRI ( $n=5-7 /$ group). Brain volume was calculated as $\mathrm{mm}^{3}$. ${ }^{*} P<0.05$. (B-D) Immunostaining for GFAP. (B) whole brain (coronal sections), (C) hippocampus (sagittal sections), and (D) amygdala (sagittal sections) are shown; images shown using $\times 4$ and $\times 60$ objectives. (E) Positive area was calculated as percentage; 2-tailed Student's $t$ test and 1-way ordinary ANOVA for multiple comparisons was applied. ${ }^{*} P<0.05 ;{ }^{* *} P<0.01$ (see Supplemental Figures 11 and 12).

IL-17 signaling contributes to alcohol-induced brain injury. Alcohol affects multiple organs and systems, including the brain and liver (4). Experimental models of alcohol dependence (CIE) and IG alcohol feeding in mice closely recapitulate pathological changes observed in patients with moderate to severe alcohol use disorder (42). In both models, the effect of increased IL-17 signaling was associated with increased astrocyte reactivity (Figure 4, A and B, and Figure 7, B-E). Indeed, systemic blockade of IL-17A reversed alcohol-induced reactive astrogliosis (characterized by proliferation, hypertrophy of astrocytes, and upregulation of astrocyte-specific markers GFAP and Slc1a3) (43), improved neuroinflammation, and reversed morphological changes associated with alcohol-induced brain injury (Figures 4 and 7). Thus, we propose that targeting IL-17A may be beneficial for treatment of alcohol addiction in patients.

Strategy for blockade of IL-17 signaling. Genetic deletion of the Il17ra gene causes increased IL-17A production in mice (44). To avoid potentially adverse effects of the disregulated cytokine network (such as upregulation of IL-17F or IL-10) $(7,45,46)$ on the alcohol drinking pattern in dependent mice, neither IL-17 $\mathrm{ra}^{-/-}$nor IL-17a $\mathrm{a}^{-/-}$mice were used for the behavioral studies. Our data are primarily based on pharmacological inhibition of IL-17 signaling. By using 2 classes of IL-17A inhibitors, either a neutralizing anti-IL-17A Ab (22) or a ROR $\gamma$ t inhibitor that prevents Th17 cell expansion but does 
A

Hippocampus

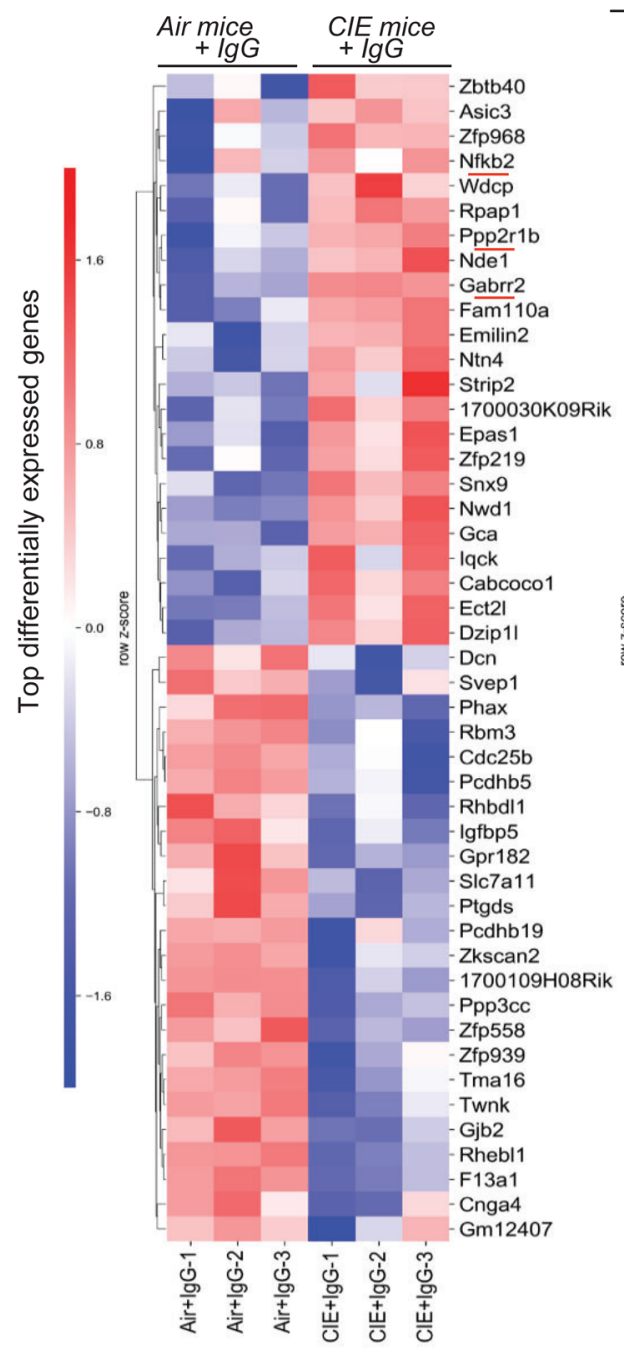

B

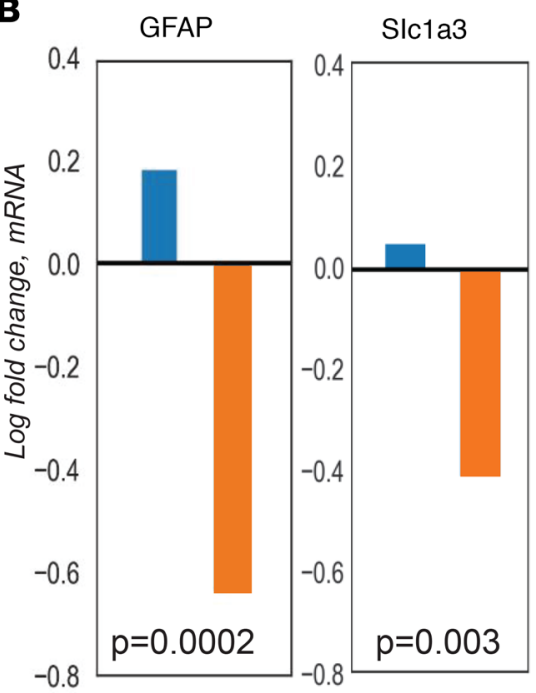

CIE vs Air + IgG

CIE mice*: Ab vs IgG

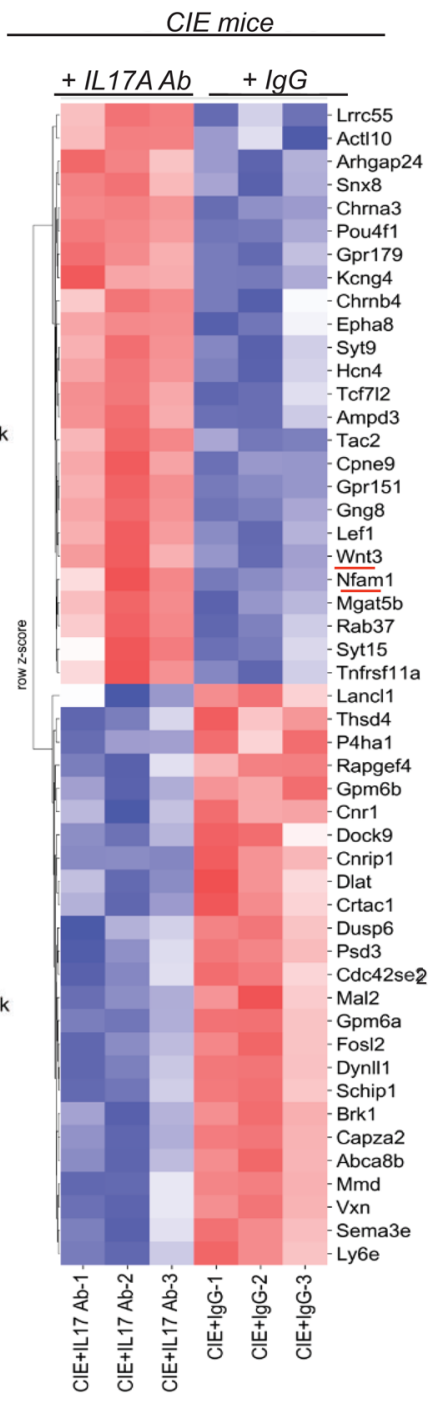

C

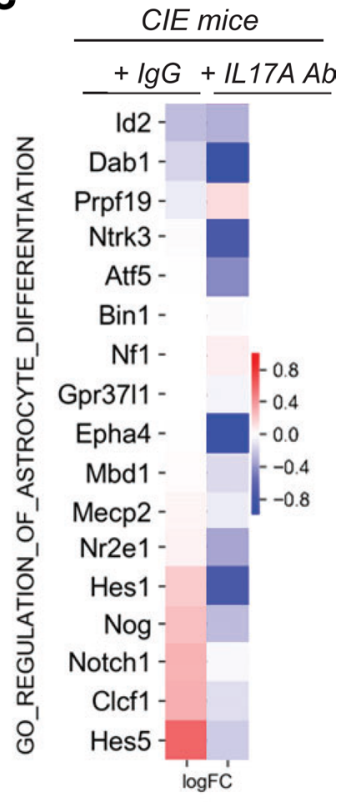

Figure 8. Blockade of IL-17A suppresses astrocyte activation in the hippocampus of CIE mice. (A) One thousand twenty alcohol dependence-associated genes were identified in the hippocampus of CIE mice + IgC versus Air mice + IgG $(P<0.05)$. Heatmaps displaying the 25 most upregulated/downregulated genes. Expression of 25 alcohol dependence-associated genes that are most significantly upregulated/downregulated in the hippocampus of CIE mice in response to anti-IL-17A Ab treatment (vs. IgG). (B) Expression of GFAP and solute carrier family 1 member 3 (SIc1a3) (C) and activation markers of hippocampal astrocytes in alcohol-dependent CIE mice treated with IgG compared with nonaddicted mice or CIE mice treated with antiIL-17A Ab compared with CIE mice treated with IgG. Log fold changes indicated by heatmap color. 
A

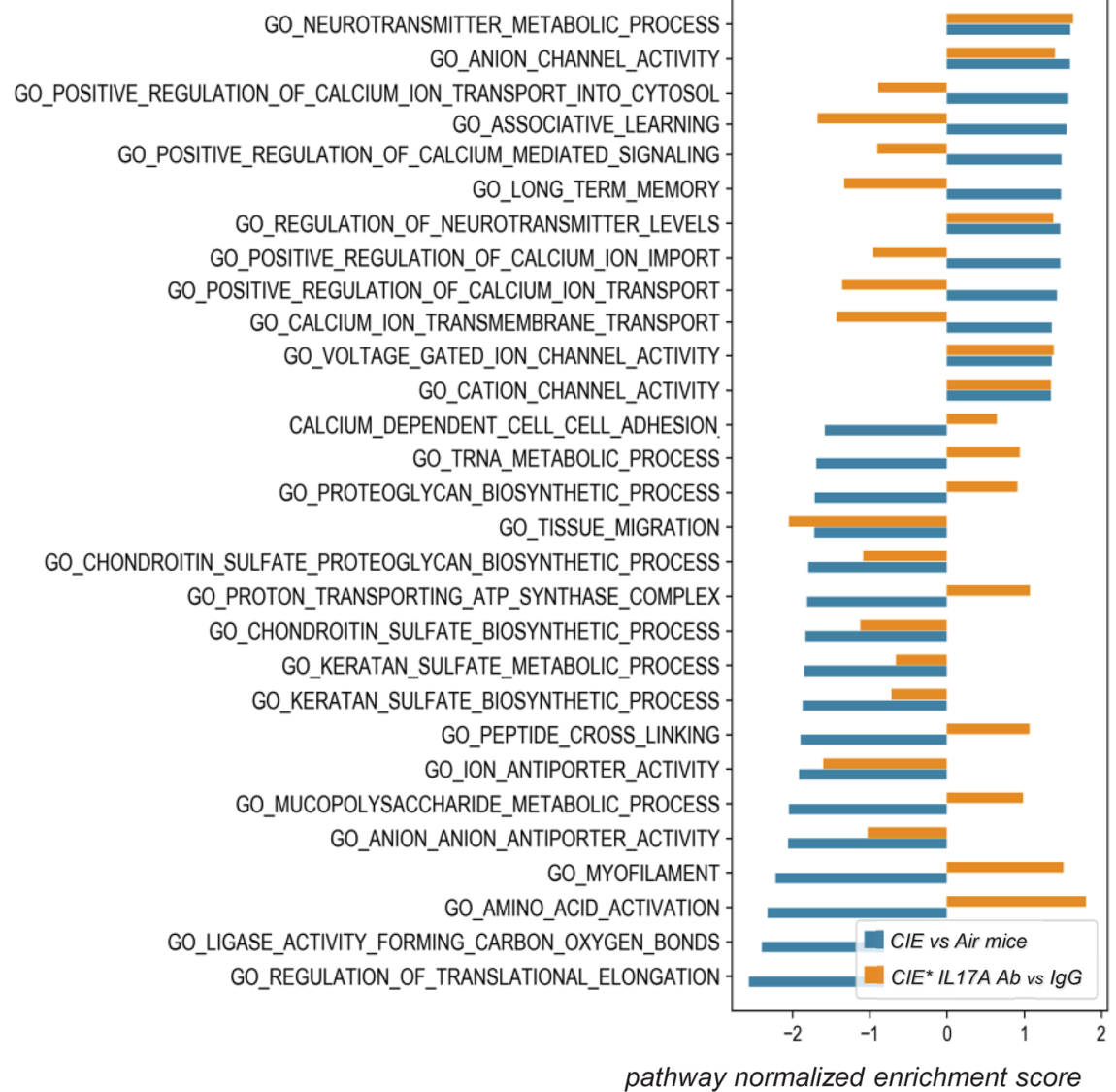

B

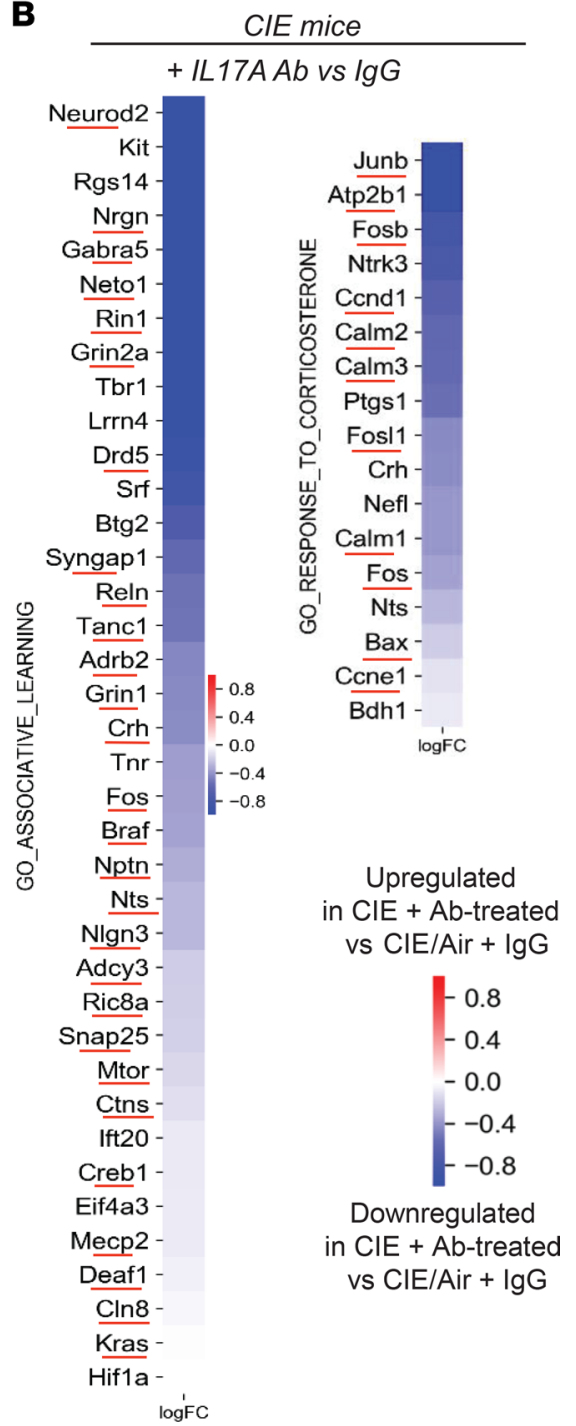

Figure 9. Blockade of IL-17A partially restores Gene Ontology terms/pathways in the hippocampus of CIE mice. (A) Selected pathways (Gene Ontology terms) were identified using gene set enrichment analysis (GSEA). Bar plots show the pathway normalized enrichment score, indicating the pathway was up- or downregulated in CIE versus Air mice (shown in blue) or in CIE IL-17A Ab versus CIE IgG mice (shown in yellow). (B) Heatmaps show individual genes' alcohol dependence-associated pathways that were restored in CIE mice upon anti-IL-17A Ab treatment, log fold changes. Receptors: Drd5 (dopamine receptor), Grin1 (glutamate receptor), Gabra5 (GABA receptor), Adrb2 (adrenergic receptor), and Htr2a and Htr3a (serotonin receptors). Hippocampus-associated learning (Neto1, corticotropin-releasing factor [CRF; Crh], Crhbp); calcium signaling (Calm 1, 2, and 3; Camk2n1 and -2; Atp2b1; Nrgn); focal adhesion (Jun, Reln, Ntrk1, Nfkb, Nfam1, Cdk5, Gsk3a and -b, Kif5b, Wnt3); cell cycle (Ccne1, Ccnd1, Kras, Rin1); apoptosis (Bax); mitochondrial function (Bdh1); regulators of transcription (Mecp2, Mbd1, Hdac2, Eftud2); metabolic (Smpd1, Hnmt, Adcy3, Ctns, Cln8). Pathway enrichment $P<0.05$ (see Supplemental Figure 13).

not affect facultatively expressing IL-17A cells, e.g., mast cells and eosinophils (23), we demonstrate that blockade of IL-17A suppresses escalation of voluntary drinking in dependent mice (Figure 6, A and B). Anti-IL-17A Ab produced an immediate but short lasting (24 hours) effect on alcohol-dependent mice. The effect of ROR $\gamma \mathrm{t}$ inhibitor was more delayed (most likely because of persistence of circulating IL-17A) but lasted for more than 2 weeks. Reduced motivation for alcohol seeking in anti-IL-17A Ab-treated, dependent mice was attributed to the IL-17A cytokine (and in part IL-17F, but not other members of the Th17 cytokine family) $(6,47)$ because anti-IL-17A Ab specifically neutralizes IL-17A homodimers and IL-17A/F heterodimers. Our study provides key preclinical evidence for targeting IL-17A in patients with alcohol addiction and compulsive alcohol drinking. Anti-IL-17 Abs and ROR $\gamma$ t inhibitors are successfully used in clinical trials for psoriasis, inflammatory bowel disease, and other autoimmune disorders, and if proven, may become a treatment of choice for alcohol dependence in patients with $\operatorname{ALD}(22,23,48)$. 
Astrocyte functions in alcohol-dependent mice. Astrocytes functionally interact with the endothelial cells and synaptic terminals of active neurons, enabling nutrient exchange between blood vessels and brain tissue and intercellular trafficking of neurotransmitters and cytokines. Astrocytes maintain extracellular homeostasis, provide support to the $\mathrm{BBB}$, regulate vascular tone and cerebral blood flow, and control synaptic neurotransmission via uptake and clearance of the major excitatory amino acid transmitters (49).

Astrocytes have been implicated in regulation of the motivational properties of alcohol, which occur on several levels (50). The acute reinforcing effects of alcohol have been shown to be mediated by the dopamine, glutamate, and GABA systems in the nucleus accumbens and amygdala. In turn, negative reinforcement via relief of negative emotional states (that also drives the motivation to seek alcohol) has been linked to activation of CRF systems in the amygdala and bed nucleus of the stria terminalis (BNST) (51). CRF-induced sensitization to alcohol withdrawal could be further enhanced by adaptive responses of astrocytes to cytokines $(50,52)$. We hypothesize that IL-17A-mediated reactive astrogliosis may facilitate focal release of IL- 6 and other cytokines to drive the brain stress systems and CRF production in the amygdala and BNST. In support of this hypothesis, systemic depletion of IL-17A reduced activation of $\mathrm{GFAP}^{+}$and $\mathrm{Slcla3}^{+}$astrocytes in CIE mice and partially restored expression of astrocyte-regulated genes (Figures 7 and 8). Thus, IL-17A affects motivational aspects of alcohol drinking behaviors in alcohol-dependent mice, and such a mechanism may be a key to reestablishing motivational homeostasis in individuals afflicted with alcohol use disorder

In summary, we demonstrate that circulating levels of IL-17A are elevated in patients with excessive alcohol use and in alcohol-dependent mice. IL-17A-dependent reactive astrogliosis is a common neuropathology in mice chronically exposed to alcohol. Development of alcohol-induced liver injury further facilitates systemic inflammation and release of IL-17A. We provide preclinical data demonstrating that blockade of IL-17A suppresses excessive alcohol drinking in alcohol-dependent mice and ameliorates alcohol-induced liver fibrosis and astrogliosis. We suggest that systemic upregulation of IL-17A is the hallmark of excessive alcohol consumption, and IL-17A might play a critical role in the pathogenesis of alcohol addiction in patients with severe alcohol use disorder.

\section{Methods}

Mice and reagents. IL-17 $\mathrm{ra}^{-1-}$ mice and WT littermates (Amgen) (53), WT mice (male C57BL/6 mice, Charles River), and WT mice (C57BL/6J mice, The Jackson Laboratories, used for 2BC experiments) were treated with anti-IL-17 Ab or IgG (CNTO 8096 and 6601, 25 mg/kg, i.p., Janssen) (22), ROR $\gamma \mathrm{t}$ inhibitor (VPR-254, $50 \mathrm{mg} / \mathrm{kg}$, oral gavage, Visionary Pharmaceuticals), or vehicle (40\% PEG400, $18 \%$ solutal in $\left.\mathrm{H}_{2} \mathrm{O}\right)$.

Experimental model of alcohol-induced liver fibrosis. IG alcohol feeding (high-cholesterol + high-fat diet [HCFD] hybrid alcohol infusion + binges) was performed as previously described (21). Mice (male C57BL/6, 12 weeks old, $n=10$ /group; data are representative of $>3$ independent experiments) were fed ad libitum diet plus a solid diet high in cholesterol and saturated fat (HCFD: 1\% w/w cholesterol, 21\% calories from lard, $17 \%$ calories from corn oil) for 2 weeks. The mice were then operated on for implantation of gastric catheters and 2 weeks later were subjected to IG feeding of liquid high-fat diet (36\% calories from corn oil) plus ethanol or isocaloric dextrose at $60 \%$ of daily caloric intake for 8 weeks for males. Ethanol dose was increased from 19 to $32 \mathrm{~g} / \mathrm{kg} / \mathrm{d}$. During the 8-week IG feeding period, mice continued to consume ad libitum HCFD to match caloric intake plus weekly binges.

Detection of serum IL-17A in patients with severe alcohol use disorder. Archived serum from healthy controls ( $n=18$, Roudebush VA Medical Center, Indianapolis) and excessive drinkers (54) (alcohol use 137-194 drinks/month $n=57$, Fairbanks Drug and Alcohol Treatment Center, Indianapolis, Indiana, USA) were analyzed by ELISA (BioLegend, 433914).

Experimental CIE-2BC model of alcohol dependence in mice. Single-housed male mice (male C57BL/6J, 10 weeks old, 4-6/group) were trained to self-administer ethanol $(15 \% v / v)$ in 2-hour 2BC sessions conducted 5 days per week (55). Upon stabilization of ethanol intake, mice were divided and exposed to either CIE (dependent mice) or Air (nondependent mice) inhalation. Weeks of CIE consisted of 4 cycles of 16 hours' intoxication/8 hours' withdrawal, followed by withdrawal from vapor for 3 days. Prior to each 16-hour intoxication, CIE mice were primed with ethanol $(1.5 \mathrm{~g} / \mathrm{kg}$, i.p., target blood alcohol level $=200$ $\mathrm{mg} / \mathrm{dL})$ and pyrazole ( $1 \mathrm{mM} / \mathrm{kg}$, i.p., to stabilize ethanol clearance rate) to inhibit alcohol dehydrogenase and prevent alcohol metabolism in the liver. Air mice were treated with pyrazole alone. 2BC resumed on 
withdrawal day 3; weeks of $2 \mathrm{BC}$ were alternated with weeks of CIE. Repeated rounds of CIE increased voluntary consumption of ethanol during $2 \mathrm{BC}$ sessions, as measured by a higher intake of CIE mice compared with Air mice during withdrawal days 3 to 7.

Gene expression profiling of murine hippocampus. Hippocampi were dissected from the brains of Air $(n=$ 3 ) and CIE \pm anti-IL-17A Ab mice ( $n=3$ /group) and analyzed by RNA-Seq. Strand-specific mRNA-sequencing libraries (polyA ${ }^{+}$) were generated using Illumina's TruSeq stranded mRNA library prep kits and sequenced on an Illumina HiSeq2500 to a depth of approximately 25 million reads. Sequencing reads were aligned to the mouse genome (National Center for Biotechnology Information [NCBI] MGSCv38, mm10) using STAR (56). Read quantification was performed with RSEM3 v1.3.0 and Ensembl annotation (Gencode v19). The R Bioconductor packages edgeR (57) and limma (58) were used to implement the limma-voom (59) method for differential expression analysis. Lowly expressed genes were filtered out (counts per million $>1$ in at least 1 sample). Trimmed mean of M-values (60) normalization was applied. The experimental design was modeled upon sample treatment (Ab or IgG) and addiction (CIE or Air). $P$ values $(P<0.05$; log fold changes were adjusted using a moderated t-statistic in limma) were calculated for each comparison. RNA-Seq data may be accessed from the NCBI's Gene Expression Omnibus database (accession number GSE137228).

Significantly enriched Gene Ontology terms were identified using GSEA (61). GSEA is a computational tool for pathway analysis that determines whether predefined pathways are significantly upregulated or downregulated between experimental conditions, without requiring an arbitrary significance cutoff on differentially expressed genes.

Statistics. Comparisons of 2 groups were analyzed using an unpaired, 2-tailed Student's $t$ test and presented as the mean $\pm \mathrm{SD}$. One-way ANOVA with a multiple comparison was used for multiple pairwise comparison among different groups. Comparisons of voluntary alcohol intake in 2BC-CIE mice are shown as the mean \pm SEM. $P<0.05$ was considered statistically significant.

Study approval. Mouse protocols were approved by the IACUC of UCSD, the University of Southern California, and the Scripps Research Institute. The human study was approved by the Indiana University Purdue University Institutional Review Board, the Research and Development Committee at Roudebush VA, and Fairbanks Drug and Alcohol Treatment Center (see Supplemental Methods).

\section{Author contributions}

JX designed the study, performed experiments, and analyzed data. HYM, XL, SR, JB, RM, MS, YK, $\mathrm{KS}, \mathrm{LS}, \mathrm{TN}$, and $\mathrm{KZ}$ performed in vitro and in vivo experiments and analyzed data. CGG, BZ, DPP, and MH provided help with histopathology analysis of the liver and brain and revised the manuscript. IM and VK performed immunophenotyping of hepatic Th17 cells. M Kreifeldt, AR, and CC performed behavioral studies in mice and analyzed the data. CC revised the manuscript. PK and SL collected and archived human samples, performed experiments, analyzed the data, and revised the manuscript. CKG, HT, BG, M Karin, DAB, and GFK provided support and critical revision of the manuscript. TK designed the study, provided support, and wrote the manuscript.

\section{Acknowledgments}

We thank K. Diggle for her excellent technical support, G. Arteel (University of Louisville Health Sciences Center) for providing lung cDNA from IG alcohol- and pair-fed mice, J. Zapf (Visionary Pharmaceuticals) for providing ROR $\gamma$ t inhibitor, M. Elloso (Janssen) for providing anti-IL-17A Ab, and Amgen for providing IL-17ra ${ }^{-/}$mice. We thank Scripps Animal Core facility (Southern California Research Center for ALPD and Cirrhosis, University of Southern California), UCSD Tissue Technology Shared Resource, and M. Budzinska (Microscopy Core, Department of Neurosciences, UCSD). We thank D. Jeste, I. Grant, and C. Achim for help and productive discussions.

This work was supported by NIH grants R01 DK101737-01A1, U01 AA022614-01A1, R01 DK09920501A1, P50AA011999, and AI043477 to TK and DAB; AI043477 to MK; NS093055 and NS054734 to BZ; R01 CA 100660 and R01 AA020864 to VK; R21AA024935, R01 DK107682, and R01 AA025208 to SL; and R21 AA024198, P60 AA006420, and R01 AA021491 to CC. Further support was received from the Herman Lopata Memorial Hepatitis Postdoctoral ALF Fellowship (to JX), 1I01CX000361 from the Veterans Affairs Research and Administration (to SL), W81XWH-12-1-0497 from the US Department of Defense, and the Showalter Scholar Award from the Ralph W. and Grace M. Showalter Research Trust (to SL). 
Address correspondence to: Tatiana Kisseleva, 9500 Gilman Drive, MC0063, UCSD, La Jolla, California 92093-0063, USA. Phone: 858.822.6022; Email: tkisseleva@ucsd.edu.

1. Koob GF, Volkow ND. Neurocircuitry of addiction. Neuropsychopharmacology. 2010;35(1):217-238

2. Le Berre AP, Fama R, Sullivan EV. Executive functions, memory, and social cognitive deficits and recovery in chronic alcoholism: a critical review to inform future research. Alcohol Clin Exp Res. 2017;41(8):1432-1443.

3. Gao B, Bataller R. Alcoholic liver disease: pathogenesis and new therapeutic targets. Gastroenterology. 2011;141(5):1572-1585.

4. Crews FT, Lawrimore CJ, Walter TJ, Coleman LG. The role of neuroimmune signaling in alcoholism. Neuropharmacology. 2017;122:56-73.

5. Ma HY, et al. The role of IL-17 signaling in regulation of the liver-brain axis and intestinal permeability in Alcoholic Liver Disease. Curr Pathobiol Rep. 2016;4(1):27-35.

6. Kolls JK, Lindén A. Interleukin-17 family members and inflammation. Immunity. 2004;21(4):467-476.

7. Meng F, et al. Interleukin-17 signaling in inflammatory, Kupffer cells, and hepatic stellate cells exacerbates liver fibrosis in mice. Gastroenterology. 2012;143(3):765-776.e3.

8. Waisman A, Hauptmann J, Regen T. The role of IL-17 in CNS diseases. Acta Neuropathol. 2015;129(5):625-637.

9. Mangan PR, et al. Transforming growth factor-beta induces development of the T(H)17 lineage. Nature. 2006;441(7090):231-234

10. Bataller R, Brenner DA. Liver fibrosis. J Clin Invest. 2005;115(2):209-218.

11. Manel N, Unutmaz D, Littman DR. The differentiation of human T(H)-17 cells requires transforming growth factor-beta and induction of the nuclear receptor RORgammat. Nat Immunol. 2008;9(6):641-649.

12. Wilson MS, et al. Bleomycin and IL-1beta-mediated pulmonary fibrosis is IL-17A dependent. J Exp Med. 2010;207(3):535-552.

13. Yoshizaki A, et al. Cell adhesion molecules regulate fibrotic process via Th1/Th2/Th17 cell balance in a bleomycin-induced scleroderma model. J Immunol. 2010;185(4):2502-2515.

14. Choi GB, et al. The maternal interleukin-17a pathway in mice promotes autism-like phenotypes in offspring. Science. 2016;351(6276):933-939.

15. Heidarianpour A, Vahidian Rezazadeh M, Zamani A. Effect of moderate exercise on serum interferon-gamma and interleukin-17 levels in the morphine withdrawal period. Int J High Risk Behav Addict. 2016;5(2):e26907.

16. Pascual M, Baliño P, Aragón CM, Guerri C. Cytokines and chemokines as biomarkers of ethanol-induced neuroinflammation and anxiety-related behavior: role of TLR4 and TLR2. Neuropharmacology. 2015;89:352-359.

17. Lemmers A, et al. The interleukin-17 pathway is involved in human alcoholic liver disease. Hepatology. 2009;49(2):646-657.

18. Lin F, et al. Alcohol dehydrogenase-specific T-cell responses are associated with alcohol consumption in patients with alcohol-related cirrhosis. Hepatology. 2013;58(1):314-324.

19. Bertola A, Mathews S, Ki SH, Wang H, Gao B. Mouse model of chronic and binge ethanol feeding (the NIAAA model). Nat Protoc. 2013;8(3):627-637.

20. Page A, et al. Alcohol directly stimulates epigenetic modifications in hepatic stellate cells. J Hepatol. 2015;62(2):388-397.

21. Lazaro R, et al. Osteopontin deficiency does not prevent but promotes alcoholic neutrophilic hepatitis in mice. Hepatology. 2015;61(1):129-140.

22. Nakajima K, et al. Distinct roles of IL-23 and IL-17 in the development of psoriasis-like lesions in a mouse model. J Immunol. 2011;186(7):4481-4489

23. Fitzpatrick LR O'Connell R, Talbott G, Bendele P, Alton G, Zapf J. A novel ROR-gamma T inhibitor (VPR-254) attenuates key parameters of innate immune colitis in mice. Gastroenterology. 2017;152: S30-S31.

24. Chen P, Miyamoto Y, Mazagova M, Lee KC, Eckmann L, Schnabl B. Microbiota and alcoholic liver disease. Alcohol Clin Exp Res. 2016;40(8):1791-1792.

25. Dubinkina VB, et al. Links of gut microbiota composition with alcohol dependence syndrome and alcoholic liver disease. Microbiome. 2017;5(1):141.

26. Peterson VL, et al. Drunk bugs: chronic vapour alcohol exposure induces marked changes in the gut microbiome in mice. Behav Brain Res. 2017;323:172-176.

27. Ivanov II, et al. Induction of intestinal Th17 cells by segmented filamentous bacteria. Cell. 2009;139(3):485-498

28. Fidler TL, et al. Intragastric self-infusion of ethanol in high- and low-drinking mouse genotypes after passive ethanol exposure. Genes Brain Behav. 2011;10(3):264-275.

29. Asatryan L, Khoja S, Rodgers KE, Alkana RL, Tsukamoto H, Davies DL. Chronic ethanol exposure combined with high fat diet up-regulates $\mathrm{P} 2 \mathrm{X} 7$ receptors that parallels neuroinflammation and neuronal loss in C57BL/6J mice. J Neuroimmunol. 2015;285:169-179.

30. Perdiguero EG, et al. The origin of tissue-resident macrophages: when an erythro-myeloid progenitor is an erythro-myeloid progenitor. Immunity. 2015;43(6):1023-1024.

31. Gomez Perdiguero E, et al. Tissue-resident macrophages originate from yolk-sac-derived erythro-myeloid progenitors. Nature. 2015;518(7540):547-551.

32. Schachtrup C, Le Moan N, Passino MA, Akassoglou K. Hepatic stellate cells and astrocytes: stars of scar formation and tissue repair. Cell Cycle. 2011;10(11):1764-1771.

33. You T, et al. IL-17 induces reactive astrocytes and up-regulation of vascular endothelial growth factor (VEGF) through JAK/ STAT signaling. Sci Rep. 2017;7:41779.

34. Hayes DM, Deeny MA, Shaner CA, Nixon K. Determining the threshold for alcohol-induced brain damage: new evidence with gliosis markers. Alcohol Clin Exp Res. 2013;37(3):425-434.

35. Pratt OE, Rooprai HK, Shaw GK, Thomson AD. The genesis of alcoholic brain tissue injury. Alcohol Alcohol. 1990;25(2-3):217-230.

36. Becker HC, Lopez MF. Increased ethanol drinking after repeated chronic ethanol exposure and withdrawal experience in C57BL/6 mice. Alcohol Clin Exp Res. 2004;28(12):1829-1838.

37. Brahmachari S, Fung YK, Pahan K. Induction of glial fibrillary acidic protein expression in astrocytes by nitric oxide. $J$ Neurosci. 
2006;26(18):4930-4939.

38. Erickson EK, Farris SP, Blednov YA, Mayfield RD, Harris RA. Astrocyte-specific transcriptome responses to chronic ethanol consumption. Pharmacogenomics J. 2018;18(4):578-589.

39. Zhou Z, Enoch MA, Goldman D. Gene expression in the addicted brain. Int Rev Neurobiol. 2014;116:251-273.

40. Heinz R, Waltenbaugh C. Ethanol consumption modifies dendritic cell antigen presentation in mice. Alcohol Clin Exp Res. 2007;31(10):1759-1771.

41. Giles DA, et al. Thermoneutral housing exacerbates nonalcoholic fatty liver disease in mice and allows for sex-independent disease modeling. Nat Med. 2017;23(7):829-838.

42. Mandrekar P, Bataller R, Tsukamoto H, Gao B. Alcoholic hepatitis: Translational approaches to develop targeted therapies. Hepatology. 2016;64(4):1343-1355.

43. Burda JE, Sofroniew MV. Reactive gliosis and the multicellular response to CNS damage and disease. Neuron. 2014;81(2):229-248.

44. Gaffen SL. Structure and signalling in the IL-17 receptor family. Nat Rev Immunol. 2009;9(8):556-567.

45. Haak S, et al. IL-17A and IL-17F do not contribute vitally to autoimmune neuro-inflammation in mice. J Clin Invest. 2009;119(1):61-69.

46. von Vietinghoff S, Ley K. IL-17A controls IL-17F production and maintains blood neutrophil counts in mice. J Immunol. 2009;183(2):865-873.

47. Starnes T, Broxmeyer HE, Robertson MJ, Hromas R. Cutting edge: IL-17D, a novel member of the IL-17 family, stimulates cytokine production and inhibits hemopoiesis. J Immunol. 2002;169(2):642-646.

48. Miossec P. Update on interleukin-17: a role in the pathogenesis of inflammatory arthritis and implication for clinical practice. RMD Open. 2017;3(1):e00284.

49. Adermark L, Bowers MS. Disentangling the role of astrocytes in alcohol use disorder. Alcohol Clin Exp Res. 2016;40(9):1802-1816

50. Breese GR, Knapp DJ, Overstreet DH, Navarro M, Wills TA, Angel RA. Repeated lipopolysaccharide (LPS) or cytokine treatments sensitize ethanol withdrawal-induced anxiety-like behavior. Neuropsychopharmacology. 2008;33(4):867-876.

51. Heilig M, Koob GF. A key role for corticotropin-releasing factor in alcohol dependence. Trends Neurosci. 2007;30(8):399-406

52. Knapp DJ, et al. Cytokine involvement in stress may depend on corticotrophin releasing factor to sensitize ethanol withdrawal anxiety. Brain Behav Immun. 2011;25(suppl 1):S146-S154.

53. Ye $\mathrm{P}$, et al. Requirement of interleukin 17 receptor signaling for lung CXC chemokine and granulocyte colony-stimulating factor expression, neutrophil recruitment, and host defense. J Exp Med. 2001;194(4):519-527.

54. National Institute on Alcohol Abuse and Alcoholism, National Institutes of Health, US Department of Health and Human Services. Helping Patients Who Drink Too Much: A Clinician's Guide: Updated 2005 Edition. Substance Abuse and Mental Health Services Administration website. https://www.integration.samhsa.gov/clinical-practice/Helping_Patients_Who_Drink_ Too_Much.pdf. Updated 2005. Accessed January 17, 2020.

55. Kreifeldt M, Le D, Treistman SN, Koob GF, Contet C. BK channel $\beta 1$ and $\beta 4$ auxiliary subunits exert opposite influences on escalated ethanol drinking in dependent mice. Front Integr Neurosci. 2013;7:105.

56. Dobin A, et al. STAR: ultrafast universal RNA-seq aligner. Bioinformatics. 2013;29(1):15-21.

57. Robinson MD, McCarthy DJ, Smyth GK. edgeR: a Bioconductor package for differential expression analysis of digital gene expression data. Bioinformatics. 2010;26(1):139-140.

58. Ritchie ME, et al. limma powers differential expression analyses for RNA-sequencing and microarray studies. Nucleic Acids Res. 2015;43(7):e47.

59. Law CW, Chen Y, Shi W, Smyth GK. voom: precision weights unlock linear model analysis tools for RNA-seq read counts. Genome Biol. 2014;15(2):R29.

60. Robinson MD, Oshlack A. A scaling normalization method for differential expression analysis of RNA-seq data. Genome Biol. 2010;11(3):R25

61. Subramanian A, et al. Gene set enrichment analysis: a knowledge-based approach for interpreting genome-wide expression profiles. Proc Natl Acad Sci U S A. 2005;102(43):15545-15550. 